\title{
A posteriori error estimates, stopping criteria, and adaptivity for multiphase compositional Darcy flows in porous media*
}

\author{
Daniele A. Di Pietro ${ }^{\dagger 1}$, Eric Flauraud ${ }^{\ddagger 2}$, Martin Vohralík ${ }^{\S 3}$, and Soleiman Yousef \} ^ { \uparrow , 4 } \\ ${ }^{1}$ University of Montpellier 2, I3M, 34057 Montpellier, France \\ ${ }^{2}$ IFP Energies nouvelles, 1 \& 4 av. Bois Préau, 92852 Rueil-Malmaison, France \\ ${ }^{3}$ INRIA Paris-Rocquencourt, B.P. 105, 78153 Le Chesnay, France \\ ${ }^{4}$ UPMC Univ. Paris 06, UMR 7598, Laboratoire Jacques-Louis Lions, 75005 Paris, France \\ \& CNRS, UMR 7598, Laboratoire Jacques-Louis Lions, 75005 Paris, France
}

April 14, 2014

\begin{abstract}
In this paper we derive a posteriori error estimates for the compositional model of multiphase Darcy flow in porous media, consisting of a system of strongly coupled nonlinear unsteady partial differential and algebraic equations. We show how to control the dual norm of the residual augmented by a nonconformity evaluation term by fully computable estimators. We then decompose the estimators into the space, time, linearization, and algebraic error components. This allows to formulate criteria for stopping the iterative algebraic solver and the iterative linearization solver when the corresponding error components do not affect significantly the overall error. Moreover, the spatial and temporal error components can be balanced by time step and space mesh adaptation. Our analysis applies to a broad class of standard numerical methods, and is independent of the linearization and of the iterative algebraic solvers employed. We exemplify it for the two-point finite volume method with fully implicit Euler time stepping, the Newton linearization, and the GMRes algebraic solver. Numerical results on two real-life reservoir engineering examples confirm that significant computational gains can be achieved thanks to our adaptive stopping criteria, already on fixed meshes, without any noticeable loss of precision.
\end{abstract}

Key words: a posteriori error analysis, adaptive algorithms, compositional Darcy flow, finite volume methods.

\section{Introduction}

Reservoir modeling is an important branch of petroleum engineering which provides predictive tools to elaborate reservoir exploration and oil production strategies. From a mathematical standpoint, the underlying models require the numerical solution of highly nontrivial problems resulting from nonlinear, strongly coupled systems of partial differential and algebraic equations. Our goal is to show that also in such complex cases, one can devise efficient solution algorithms based on a posteriori error estimates that ensure error control and allow significant computational savings in numerical simulations. Improving the performance of reservoir simulators is a key point, since the simulation of complex Darcian flows in three space dimensions accounts for the largest part of the computational effort in optimization models for petroleum fields exploitation.

We focus on the Darcy flow of several fluids through a subsurface porous medium. We suppose that the fluids are composed of a finite number of components that constitute the phases in the reservoir. Under

\footnotetext{
*This research was supported by the ERT project "Enhanced oil recovery and geological sequestration of $\mathrm{CO}_{2}$ : mesh adaptivity, a posteriori error control, and other advanced techniques" (LJLL/IFPEN).

taniele.di-pietro@univ-montp2.fr

teric.flauraud@ifpen.fr

$\S$ martin.vohralik@inria.fr

"Corresponding author, yousef@ann.jussieu.fr
} 
the assumption that the flow process is isothermal, the equations that govern the compositional model are the conservation of the amount of each component supplemented by algebraic equations expressing the conservation of volume, the conservation of the quantity of matter, and the thermodynamic equilibrium.

Several numerical methods have been proposed for the discretization of the compositional model. Finite difference and finite element methods can be used under some assumptions on the physical data, see [4, 10, 26, 70] and references therein, but it may be difficult to recover local mass conservation therein. Mixed finite element methods do not suffer from such a drawback and have been extensively used and analyzed, see, e.g. [30, 24, 25] and references therein.

Finite volume methods have long been popular in reservoir engineering owing to their numerous advantages: they meet the industrial constraints of robustness and low computational cost, they satisfy local conservation, are simple to code, and can be used on a large variety of meshes. Several nodecentered finite volume discretizations are presented and compared by Huber and Helming [45] and a recent study is given in Eymard et al. 39]. Cell-centered finite volume methods have been considered in [54, 66, 38, 40. A symmetric and coercive cell-centered finite volume scheme for discretizing Darcy fluxes has been proposed in [6]. A particularly popular family of cell-centered finite volume schemes in the oil industry is that of multi-point methods, which can be easily plugged into traditional simulators thereby allowing to handle very complicated geometries. They have been studied in the multiphase compositional context by Aavatsmark et al. 1, 2, see also the references therein. More recently, a variation with compact stencil and increased stability has been proposed and analyzed in [5]. For an up-to-date review of discretization methods for diffusive fluxes in the context of geoscience models we refer to [28]; see also Droniou [31] for a wider-scope introduction to finite volume methods for diffusive problems on general meshes.

To the best of our knowledge, almost no work has been done to this day on a posteriori error estimates and stopping criteria for the general version of the multiphase compositional model allowing an arbitrary number of phases and components. The goal of the present work is to fill this gap.

A posteriori error estimates enable to monitor the computational error. For model unsteady nonlinear problems, some of the first rigorous results were obtained by Eriksson and Johnson 34 and by Verfürth 64, 65]. Degenerate problems have subsequently been studied by Nochetto et al. [56], Ohlberger [57, and lastly in 29 . An adaptation of the estimators for finite volume discretizations of hyperbolic conservation laws of Kröner and Ohlberger [50] to a steam-assisted gravity drainage two-component, three-phase flow has been presented by Mamaghani et al. [55. For multiphase reservoir simulation, dynamic local grid refinement was first considered by Heinemann [42] and Ewing et al. [37. Then, in [61, Sammon discussed the development of adaptive techniques in the context of unstructured grids for compositional simulation. Local refinement based on structured grid adaptive mesh refinement was probably first applied by Hornung and Trangenstein in [44] and Trangenstein and Bi 63. In Pau et al. [58, 59], another development of a structured grid adaptive mesh refinement algorithm for incompressible/compressible two-phase flow in porous media is discussed. Recently, first rigorous results for immiscible incompressible two-phase flow have appeared. Reference 68 develops a general abstract framework for a posteriori estimates of the dual norm of the residual augmented by a nonconformity evaluation term, and proposes an adaptive algorithm with stopping criteria for the iterative solution of the arising linear systems and iterative linearization/iterative coupling, wherein the spatial and temporal errors are equilibrated. This leads to both error control and important computational savings. Rigorous energy-spaces-type bounds have then been obtained for vertex-centered finite volume discretizations in 20].

In this paper we derive fully computable a posteriori error estimates for a general version of the multiphase compositional model. Following [68], the results are derived for the error measured as the dual norm of the residual augmented by a nonconformity evaluation term. This error measure stems directly from the given model and simplifies the analysis. It has recently been proved, for conforming discretizations of model nonlinear problems such as the immiscible incompressible two-phase flow in [20] and of the two-phase Stefan problem in [29], that this error measure is an upper bound for an energyspaces-type norm of the difference between the exact and approximate solutions.

Our a posteriori error estimate can be separated into parts identifying the various sources of the error in the numerical solution. More specifically, we construct: a spatial estimator incorporating the errors related to the space discretization and to the nonconformity of the scheme; a temporal estimator accounting for the time discretization error; a linearization estimator due to the approximate linearization; and, finally, an algebraic estimator due to the inexact solution of the arising linear algebraic systems. Distinguishing the different error components allows to formulate stopping criteria for 
the iterative linearization and iterative algebraic solvers that halt the iterations when the corresponding error components no longer affect significantly the overall error. We also propose to equilibrate the space and time errors by adapting the choice of the time step and adjusting adaptively the computational mesh. These criteria are collected to design an adaptive algorithm for the resolution of the multiphase compositional model ensuring a user-given precision and significant computational savings compared to the classical resolution of the model, and this already on fixed meshes. Additionally, our estimators prove capable of identifying relevant features of the solution such as well singularities and moving fronts. This anticipates them to be a good tool for the local adaptation of the spatial mesh. This topic will be treated in detail in a future work.

The paper is organized as follows. In Section 2 we describe the system of equations for the multiphase compositional model and identify the unknowns and relevant physical properties along with their dependencies. We also discuss therein a fully implicit cell-centered finite volume discretization with phase upwind and two-point discretization of the diffusive fluxes. In Section 3 we introduce the corresponding weak formulation, define the error measure, and state our a posteriori error estimate. In Section 4 we distinguish the different arising error components and propose a fully adaptive algorithm. Finally, in Section 5 we illustrate our theoretical analysis by numerical results; already on fixed meshes, we obtain the same precision and a speed-up factor of order 10 in terms of the total number of algebraic solver iterations in comparison with the classical resolution.

\section{Setting}

We introduce in this section the multiphase compositional model and its finite volume discretization.

\subsection{The multiphase compositional model}

The compositional Darcy model describes the flow of several fluids through a porous medium reservoir occupying the space region $\Omega \subset \mathbb{R}^{d}, d \in\{2,3\}$, over the time interval $\left(0, t_{\mathrm{F}}\right), t_{\mathrm{F}}>0$. We assume that $\Omega$ is a bounded connected polygon if $d=2$ or polyhedron if $d=3$ and follows essentially [26, 39].

\subsubsection{Model unknowns}

We consider a system where matter is present in different phases collected in the set $\mathcal{P}=\{p\}$, each containing one or more components from the set $\mathcal{C}=\{c\}$. For a given phase $p \in \mathcal{P}$, let $\mathcal{C}_{p} \subset \mathcal{C}$ be the set of its components, and, for a given component $c \in \mathcal{C}$, denote by $\mathcal{P}_{c}$ the set of the phases which contain c. For a given phase $p \in \mathcal{P}, S_{p}$ denotes the saturation, i.e., the fraction of the pore volume occupied by $p$, and, for each component $c \in \mathcal{C}_{p}, C_{p, c}$ is the corresponding molar fraction in $p$. Saturations are collected in the vector $\boldsymbol{S}=\left(S_{p}\right)_{p \in \mathcal{P}}$ while, for all $p \in \mathcal{P}$, molar fractions are collected in the vectors $\boldsymbol{C}_{p}:=\left(C_{p, c}\right)_{c \in \mathcal{C}_{p}}$. We tackle here the isothermal case where no energy source or sink is present and the temperature of both the fluids and the porous medium are fixed to a given value. The dependence on the temperature is hence not taken into account in what follows. We denote by $P$ the reference pressure such that the phase pressures $P_{p}, p \in \mathcal{P}$, are expressed as

$$
P_{p}=P_{p}(P, \boldsymbol{S}):=P+P_{\mathrm{c}_{p}}(\boldsymbol{S}),
$$

where $P_{\mathrm{c}_{p}}(\boldsymbol{S})$ is a generalized capillary pressure. In a two-phase system, the standard capillary pressure is defined as the difference between the non-wetting and wetting phase pressures. In multiphase systems, capillary pressures are usually obtained by combining the expressions of capillary pressures for each couple of non-wetting and wetting phases. Formula 2.1 allows to deal with this aspect in a more abstract and mathematically convenient way by introducing a symmetry in the treatment of the phases. In practice, the reference pressure is chosen as the pressure of a suitable phase $p \in \mathcal{P}$, whose generalized capillary pressure is hence identically zero. The unknowns of the model are collected in the vector

$$
\mathcal{X}:=\left(\begin{array}{c}
P \\
\left(S_{p}\right)_{p \in \mathcal{P}} \\
\left(C_{p, c}\right)_{p \in \mathcal{P}, c \in \mathcal{C}_{p}}
\end{array}\right) .
$$

This gives a total of $1+N_{\mathcal{P}}+\sum_{p \in \mathcal{P}} N_{\mathcal{C}_{p}}$ unknowns, (here and in what follows, $N_{\mathcal{S}}$ stands for the cardinality of the set $\mathcal{S}$ ). 


\subsubsection{Physical properties}

The porous medium is characterized by its porosity $\phi$ and its absolute permeability $\boldsymbol{\Lambda}$, both of which are assumed constant in time for the sake of simplicity. For each fluid phase $p \in \mathcal{P}$, the following properties are relevant to the model (the usual dependence is provided in brackets): (i) the molar density $\zeta_{p}\left(P_{p}, \boldsymbol{C}_{p}\right)$; (ii) the mass density $\rho_{p}\left(P_{p}, \boldsymbol{C}_{p}\right)$; (iii) the viscosity $\mu_{p}\left(P_{p}, \boldsymbol{C}_{p}\right)$; (iv) the relative permeability $k_{\mathrm{r}, p}(\boldsymbol{S})$; (v) for all $c \in \mathcal{C}_{p}$, the fugacity $f_{c, p}\left(P_{p}, \boldsymbol{C}_{p}\right)$. It is also convenient to define for each phase $p \in \mathcal{P}$ the mobility given by $\nu_{p}\left(P_{p}, \boldsymbol{S}, \boldsymbol{C}_{p}\right):=\zeta_{p}\left(P_{p}, \boldsymbol{C}_{p}\right) \frac{k_{\mathrm{r}, p}(\boldsymbol{S})}{\mu_{p}\left(P_{p}, \boldsymbol{C}_{p}\right)}$.

\subsubsection{Governing partial differential equations}

The governing partial differential equations (PDEs) are obtained by enforcing the conservation of the amounts of each component, using a constitutive law to relate the average phase velocities to the unknowns of the model. The conservation of the amount of each component is expressed by the following system of $N_{\mathcal{C}}$ PDEs:

$$
\partial_{t} l_{c}+\nabla \cdot \boldsymbol{\Phi}_{c}=q_{c}, \quad \forall c \in \mathcal{C},
$$

where, for each $c \in \mathcal{C}$, the component flux $\boldsymbol{\Phi}_{c}$ has the following expression:

$$
\boldsymbol{\Phi}_{c}:=\sum_{p \in \mathcal{P}_{c}} \boldsymbol{\Phi}_{p, c}, \quad \boldsymbol{\Phi}_{p, c}=\boldsymbol{\Phi}_{p, c}\left(P_{p}, \boldsymbol{S}, \boldsymbol{C}_{p}\right):=\nu_{p}\left(P_{p}, \boldsymbol{S}, \boldsymbol{C}_{p}\right) C_{p, c} \boldsymbol{v}_{p}\left(P_{p}, \boldsymbol{C}_{p}\right),
$$

and for all $p \in \mathcal{P}, \boldsymbol{v}_{p}$ represents the average phase velocity given by Darcy's law,

$$
\boldsymbol{v}_{p}=\boldsymbol{v}_{p}\left(P_{p}, \boldsymbol{C}_{p}\right)=-\boldsymbol{\Lambda}\left(\nabla P_{p}-\rho_{p}\left(P_{p}, \boldsymbol{C}_{p}\right) \boldsymbol{g}\right)=-\boldsymbol{\Lambda}\left(\nabla P_{p}+\rho_{p}\left(P_{p}, \boldsymbol{C}_{p}\right) g \nabla z\right),
$$

where $\boldsymbol{g}$ denotes the gravity vector acting in the negative $z$ direction and $g$ its Euclidian norm. Additionally, in $(2.2), q_{c} \in L^{2}\left(\left(0, t_{\mathrm{F}}\right) ; L^{2}(\Omega)\right)$ denotes a source or sink and $l_{c}$ is the amount (in moles) of component $c$ per unit volume,

$$
l_{c}=l_{c}(\mathcal{X})=\phi \sum_{p \in \mathcal{P}_{c}} \zeta_{p}\left(P_{p}, \boldsymbol{C}_{p}\right) S_{p} C_{p, c}
$$

For the sake of simplicity, we assume that no-flow boundary conditions are prescribed for all the component fluxes,

$$
\boldsymbol{\Phi}_{c} \cdot \boldsymbol{n}_{\Omega}=0 \quad \text { on } \partial \Omega \times\left(0, t_{\mathrm{F}}\right) \quad \forall c \in \mathcal{C},
$$

where $\partial \Omega$ denotes the boundary of $\Omega$ and $\boldsymbol{n}_{\Omega}$ its unit outward normal. At $t=0$ we prescribe the initial amount of each component,

$$
l_{c}(\cdot, 0)=l_{c}^{0} \quad \forall c \in \mathcal{C} .
$$

\subsubsection{Closure algebraic equations}

The governing PDEs of the previous section need to be supplemented by a system of algebraic equations imposing the volume conservation, the conservation of the quantity of matter, and local thermodynamic equilibria. First, it is assumed that the pore volume is saturated by the phases, i.e.,

$$
\sum_{p \in \mathcal{P}} S_{p}=1
$$

Next, by definition, the molar fractions satisfy

$$
\sum_{c \in \mathcal{C}_{p}} C_{p, c}=1 \quad \forall p \in \mathcal{P},
$$

which corresponds to a total of $N_{\mathcal{P}}$ algebraic equations. Finally, we assume the thermodynamic equilibrium expressed by

$$
\sum_{c \in \mathcal{C}}\left(N_{\mathcal{P}_{c}}-1\right)=\sum_{p \in \mathcal{P}} N_{\mathcal{C}_{p}}-N_{\mathcal{C}}
$$

equalities of fugacities. Formulating the thermodynamic equilibrium 2.10 for an arbitrary number of phases and components lies out of the scope of the present work, and we limit ourselves in the next section to two examples. For further details we refer to Bear [14] or Chen et al. 25]. 


\subsubsection{Examples}

To fix the ideas, we now present two common examples of multiphase compositional flows in the context of reservoir simulation.

Example 1 (Three-phase flow). We consider three phases, typically water, gas, and oil, $\mathcal{P}=\{\mathrm{w}, \mathrm{g}, \mathrm{o}\}$, containing $N_{\mathcal{C}}$ components decomposed into $N_{\mathcal{H}}:=N_{\mathcal{C}}-1$ hydrocarbon components from the set $\mathcal{H}$, and one water component $\mathrm{e}$. Usually, under isothermal conditions, mass interchange occurs only between the gas phase and the oil phase. Thus, the water phase contains only the water component e with molar fraction $C_{\mathrm{w}, \mathrm{e}}=1$. The equations from 2.10 expressing the thermodynamic equilibrium between the oil and gas phases take here the form

$$
f_{c, \mathrm{o}}\left(P, \boldsymbol{C}_{\mathrm{o}}\right)=f_{c, \mathrm{~g}}\left(P, \boldsymbol{C}_{\mathrm{g}}\right), \quad \forall c \in \mathcal{H},
$$

which corresponds to $N_{\mathcal{H}}$ algebraic equations. Condition (2.11) is often reformulated as

$$
K_{c}^{\mathrm{o}}\left(P, \boldsymbol{C}_{\mathrm{o}}\right) C_{\mathrm{o}, c}=K_{c}^{\mathrm{g}}\left(P, \boldsymbol{C}_{\mathrm{g}}\right) C_{\mathrm{g}, c}, \quad \forall c \in \mathcal{H},
$$

where $K_{c}^{\mathrm{o}}, K_{c}^{\mathrm{g}}$ are the so-called equilibrium constants for the component $c \in \mathcal{H}$ in the oil and gas phases, respectively. Letting $K_{c}^{\mathrm{o}, \mathrm{g}}:=\frac{K_{c}^{\circ}}{K_{c}^{\mathrm{g}}}$, we can write 2.11) as

$$
C_{\mathrm{g}, c}=K_{c}^{\mathrm{o}, \mathrm{g}}\left(P, C_{\mathrm{o}}, C_{\mathrm{g}}\right) C_{\mathrm{o}, c}, \quad \forall c \in \mathcal{H},
$$

with $K_{c}^{\mathrm{o}, \mathrm{g}}$ the equilibrium constant between the oil and gas phases for the component $c \in \mathcal{H}$. Using the equations of mass conservation (2.2), volume conservation (2.8), conservation of the quantity of matter 2.9), and the thermodynamic equilibrium 2.13, the three-phase compositional model reads

$$
\begin{aligned}
& \partial_{t}\left(\phi \zeta_{\mathrm{w}} S_{\mathrm{w}} C_{\mathrm{w}, \mathrm{e}}\right)+\nabla \cdot\left(\frac{\zeta_{\mathrm{w}} k_{\mathrm{r}, \mathrm{w}}}{\mu_{\mathrm{w}}} C_{\mathrm{w}, \mathrm{e}} \boldsymbol{v}_{\mathrm{w}}\right)=q_{\mathrm{e}}, \\
& \partial_{t}\left(\phi\left(\zeta_{\mathrm{o}} S_{\mathrm{o}} C_{\mathrm{o}, c}+\zeta_{\mathrm{g}} S_{\mathrm{g}} C_{\mathrm{g}, c}\right)\right)+\nabla \cdot\left(\frac{\zeta_{\mathrm{o}} k_{\mathrm{r}, \mathrm{o}}}{\mu_{\mathrm{o}}} C_{\mathrm{o}, c} \boldsymbol{v}_{\mathrm{o}}+\frac{\zeta_{\mathrm{g}} k_{\mathrm{r}, \mathrm{g}}}{\mu_{\mathrm{g}}} C_{\mathrm{g}, c} \boldsymbol{v}_{\mathrm{g}}\right)=q_{c} \quad \forall c \in \mathcal{H}, \\
& S_{\mathrm{w}}+S_{\mathrm{o}}+S_{\mathrm{g}}=1, \\
& C_{\mathrm{w}, \mathrm{e}}=1 \text {, } \\
& \sum_{c \in \mathcal{C}_{\mathrm{o}}} C_{\mathrm{o}, c}=1, \\
& \sum_{c \in \mathcal{C}_{\mathrm{g}}} C_{\mathrm{g}, c}=1, \\
& K_{c}^{\mathrm{o}, \mathrm{g}}\left(P, \boldsymbol{C}_{\mathrm{o}}, \boldsymbol{C}_{\mathrm{g}}\right) C_{\mathrm{o}, c}=C_{\mathrm{g}, c}, \quad \forall c \in \mathcal{H},
\end{aligned}
$$

where the phase pressures are given by 2.1) and the Darcy velocities by 2.4., while the boundary and initial conditions are respectively specified by (2.6) and (2.7). The total number of equations is $2 N_{\mathcal{C}}+3$. Recall that the unknowns are one reference pressure, $N_{\mathcal{P}}$ saturations, and $\sum_{p \in \mathcal{P}} N_{\mathcal{C}_{p}}$ molar fractions, totaling

$$
1+N_{\mathcal{P}}+\sum_{p \in \mathcal{P}} N_{\mathcal{C}_{p}}=1+3+\left(1+2 \times\left(N_{\mathcal{C}}-1\right)\right)=2 N_{\mathcal{C}}+3,
$$

which gives us the same number of equations as unknowns.

Example 2 (Miscible two-phase flow). We next examine how the model of Example 1 simplifies when water is not present. This is precisely the case considered in the numerical examples of Section 5 below. The phases are now gas and oil, corresponding to $\mathcal{P}=\{\mathrm{g}, \mathrm{o}\}$, composed of $N_{\mathcal{C}}$ hydrocarbon components with, using the notation of Example 1. $\mathcal{C}=\mathcal{H}$. Mass interchange is allowed between these two phases, and the thermodynamic equilibrium relations are given by (2.11) or 2.12) as in the previous example. 
The system of equations 2.14 simplifies to

$$
\begin{aligned}
\partial_{t}\left(\phi\left(\zeta_{\mathrm{o}} S_{\mathrm{o}} C_{\mathrm{o}, c}+\zeta_{\mathrm{g}} S_{\mathrm{g}} C_{\mathrm{g}, c}\right)\right)+\nabla \cdot\left(\frac{\zeta_{\mathrm{o}} k_{\mathrm{r}, \mathrm{o}}}{\mu_{\mathrm{o}}} C_{\mathrm{o}, c} \boldsymbol{v}_{\mathrm{o}}+\frac{\zeta_{\mathrm{g}} k_{\mathrm{r}, \mathrm{g}}}{\mu_{\mathrm{g}}} C_{\mathrm{g}, c} \boldsymbol{v}_{\mathrm{g}}\right) & =q_{c}, \quad \forall c \in \mathcal{C}, S, \\
S_{\mathrm{o}}+S_{\mathrm{g}} & =1, \\
\sum_{c \in \mathcal{C}_{\mathrm{o}}} C_{\mathrm{o}, c} & =1, \\
\sum_{c \in \mathcal{C}_{\mathrm{g}}} C_{\mathrm{g}, c} & =1, \\
K_{c}^{\mathrm{o}, \mathrm{g}}\left(P, C_{\mathrm{o}}, C_{\mathrm{g}}\right) C_{\mathrm{o}, c} & =C_{\mathrm{g}, c}, \quad \forall c \in \mathcal{C},
\end{aligned}
$$

amounting to $2 N_{\mathcal{C}}+3$ equations. Also in this case we have the same number of equations as unknowns, the latter equaling to

$$
1+N_{\mathcal{P}}+\sum_{p \in \mathcal{P}} N_{\mathcal{C}_{p}}=1+2+\left(2 \times\left(N_{\mathcal{C}}\right)\right)=2 N_{\mathcal{C}}+3 .
$$

\subsection{An implicit finite volume scheme with phase-upwind and two-point dis- cretization of diffusive fluxes}

In this section we briefly discuss a fully implicit numerical scheme for the multiphase compositional model of Section 2.1 based on phase-upwind and two-point finite volume discretization of diffusive fluxes. The use of phase-upwind for the finite volume discretization of the Darcy problem is considered, e.g., in Brenier and Jaffré [16] and Eymard et al. [0]. This scheme is of primary importance due to its stability and consequent popularity in the oil industry.

\subsubsection{Space-time meshes}

Let $\left(\tau_{n}\right)_{1 \leq n \leq N}$ denote a sequence of positive real numbers corresponding to the discrete time steps such that $t_{\mathrm{F}}=\sum_{n=1}^{N} \tau_{n}$. We consider the discrete times $\left(t^{n}\right)_{0 \leq n \leq N}$ such that $t^{0}:=0$ and, for $1 \leq n \leq N$, $t^{n}:=\sum_{i=1}^{n} \tau_{i}$; then we define the time intervals $I_{n}:=\left(t^{n-1}, t^{n}\right)$. For a function of time $v$ with sufficient regularity we denote $v^{n}:=v\left(t^{n}\right), 0 \leq n \leq N$, and, for $1 \leq n \leq N$, we define the backward differencing operator

$$
\partial_{t}^{n} v:=\frac{1}{\tau^{n}}\left(v^{n}-v^{n-1}\right)
$$

that we shall use for both scalar and vector functions.

Let $\left(\mathcal{M}^{n}\right)_{0 \leq n \leq N}$ denote a family of meshes of the space domain $\Omega$ superadmissible in the sense of Eymard et al. [38, Definition 3.1]. Common instances of superadmissible meshes are Cartesian orthogonal grids or matching simplicial meshes that satisfy the (strict) Delaunay condition. Superadmissibility requires, in particular, that for all $M \in \mathcal{M}^{n}$ there exists a point $\boldsymbol{x}_{M} \in M$ (the cell center), and for all mesh faces $\sigma$, there exists a point $\overline{\boldsymbol{x}}_{\sigma} \in \sigma$ (the face center) such that, for all faces $\sigma$ lying on the boundary of an element $M$, the line segment joining $\boldsymbol{x}_{M}$ with $\overline{\boldsymbol{x}}_{\sigma}$ is $\boldsymbol{\Lambda}^{-1}$-orthogonal to $\sigma$. In what follows we let, for all $M \in \mathcal{M}^{n}$ and all $\sigma \in \mathcal{E}_{M}^{\mathrm{i}, n}, d_{M, \sigma}:=\operatorname{dist}\left(\boldsymbol{x}_{M}, \overline{\boldsymbol{x}}_{\sigma}\right)$, where $\mathcal{E}_{M}^{\mathrm{i}, n}$ denotes the faces of an element $M \in \mathcal{M}^{n}$ not lying on $\partial \Omega$. For every element $M \in \mathcal{M}^{n}$, we denote by $|M|$ its $d$-dimensional Lebesgue measure and by $h_{M}$ its diameter. For $0 \leq n \leq N$, we denote by $\mathcal{E}^{n}$ the set of mesh faces. Boundary faces are collected in the set $\mathcal{E}^{\mathrm{b}, n}:=\left\{\sigma \in \mathcal{E}^{n} ; \sigma \subset \partial \Omega\right\}$ and we let $\mathcal{E}^{\mathrm{i}, n}:=\mathcal{E}^{n} \backslash \mathcal{E}^{\mathrm{b}, n}$. For an internal face $\sigma \in \mathcal{E}^{\mathrm{i}, n}$ we fix an arbitrary orientation and denote the corresponding unit normal vector by $\boldsymbol{n}_{\sigma}$. For a boundary face $\sigma \in \mathcal{E}^{\mathrm{b}, n}, \boldsymbol{n}_{\sigma}$ coincides with the exterior unit normal $\boldsymbol{n}_{\Omega}$ of $\Omega$.

\subsubsection{Finite volume discretization}

In the context of cell-centered finite volume methods, the unknowns of the model are discretized using one value per cell: For all $0 \leq n \leq N$ we let

$$
\mathcal{X}_{\mathcal{M}}^{n}:=\left(\mathcal{X}_{M}^{n}\right)_{M \in \mathcal{M}^{n}}, \quad \mathcal{X}_{M}^{n}:=\left(\begin{array}{c}
P_{M}^{n} \\
\left(S_{p, M}^{n}\right)_{p \in \mathcal{P}} \\
\left(C_{p, c, M}^{n}\right)_{p \in \mathcal{P}, c \in \mathcal{C}_{p}}
\end{array}\right) \quad \forall M \in \mathcal{M}^{n}
$$


In particular, in practice, the initial condition (2.7) needs to be augmented to

$$
\mathcal{X}_{\mathcal{M}}(\cdot, 0)=\mathcal{X}_{\mathcal{M}}^{0}
$$

where $\mathcal{X}_{\mathcal{M}}^{0}$ typically results from a steady-state equilibrium computation. For simplicity, we suppose that $l_{c}^{0}$ in 2.7) is piecewise constant on $\mathcal{M}^{0}$ and exactly satisfied by the corresponding components of $\mathcal{X}_{\mathcal{M}}^{0}$. For all time steps $0 \leq n \leq N$ and all $M \in \mathcal{M}^{n}$, the discrete phase saturations are collected in the vector $S_{M}^{n}:=\left(S_{p, M}^{n}\right)_{p \in \mathcal{P}}$ while, for all $p \in \mathcal{P}$, the discrete molar fractions are collected in the vector $C_{p, M}^{n}:=\left(C_{p, c, M}^{n}\right)_{c \in \mathcal{C}_{p}}$. We consider in what follows an isotropic, possibly heterogeneous medium such that the local (cell) permeability tensor satisfies $\left.\boldsymbol{\Lambda}\right|_{M}=\lambda_{M} \boldsymbol{I} \boldsymbol{d}$ for all $M \in \mathcal{M}^{n}$ and a scalar $\lambda_{M}>0$. Since we consider superadmissible meshes, this assumption ensures the consistency of the two-point finite volume discretization of diffusive fluxes. We observe, in passing, that the consistency of the discretization scheme is not required in the a posteriori error analysis in the sense that the estimate of Theorem 3.3 holds true even if consistency does not, and its proof does not require to specify the origin of the discrete approximation. This property may have some interest in practice when superadmissibility cannot be guaranteed, since a consistency estimator could be devised. Of course, in practice, we advocate the systematic use of unconditionally consistent schemes.

For each phase $p \in \mathcal{P}$, the corresponding phase pressure inside each cell $M \in \mathcal{M}^{n}$ at time step $0 \leq n \leq N$ is given by

$$
P_{p, M}^{n}=P_{p, M}^{n}\left(P_{M}^{n}, \boldsymbol{S}_{M}^{n}\right):=P_{M}^{n}+P_{\mathrm{c}_{p}}\left(\boldsymbol{S}_{M}^{n}\right) .
$$

The PDEs 2.2 expressing the conservation of the amount of each component are discretized as follows: For all $1 \leq n \leq N$, we require

$$
|M| \partial_{t}^{n} l_{c, M}+\sum_{\sigma \in \mathcal{E}_{M}^{\mathrm{i}, n}} F_{c, M, \sigma}\left(\mathcal{X}_{\mathcal{M}}^{n}\right)=|M| q_{c, M}^{n}, \quad \forall c \in \mathcal{C}, \forall M \in \mathcal{M}^{n}
$$

where $q_{c, M}^{n}:=\int_{I_{n}} \int_{M} q_{c} /\left(|M| \tau_{n}\right) \mathrm{d} \mathbf{x d} t$ (more details about the source term will be given in the numerical tests), and the accumulation term is given, for all $0 \leq n \leq N$, by the following discrete version of (2.5):

$$
l_{c, M}^{n}=l_{c, M}\left(\mathcal{X}_{M}^{n}\right):=\phi \sum_{p \in \mathcal{P}_{c}} \zeta_{p}\left(P_{p, M}^{n}, C_{p, M}^{n}\right) S_{p, M}^{n} C_{p, c, M}^{n} \quad \forall c \in \mathcal{C}, \forall M \in \mathcal{M}^{n}
$$

For each component $c \in \mathcal{C}$, its total flux across $\sigma$ results from the sum of the corresponding fluxes for each phase $p \in \mathcal{P}_{c}$, i.e.,

$$
F_{c, M, \sigma}\left(\mathcal{X}_{\mathcal{M}}^{n}\right):=\sum_{p \in \mathcal{P}_{c}} F_{p, c, M, \sigma}\left(\mathcal{X}_{\mathcal{M}}^{n}\right)
$$

where, for all $p \in \mathcal{P}_{c}$, all $M \in \mathcal{M}^{n}$, and all $\sigma \in \mathcal{E}_{M}^{\mathrm{i}, n}$ with $\sigma=\partial M \cap \partial L$,

$$
F_{p, c, M, \sigma}\left(\mathcal{X}_{\mathcal{M}}^{n}\right)=\nu_{p}^{\uparrow}\left(\mathcal{X}_{\mathcal{M}}^{n}\right) C_{p, c, M_{p}^{\uparrow}}^{n} F_{p, M, \sigma}\left(\mathcal{X}_{\mathcal{M}}^{n}\right), \quad M_{p}^{\uparrow}= \begin{cases}M & \text { if } P_{p, M}^{n}-P_{p, L}^{n} \geq 0 \\ L & \text { otherwise }\end{cases}
$$

and with $C_{p, c, M_{p}^{\uparrow}}^{n}$ and $\nu_{p}^{\uparrow}\left(\mathcal{X}_{\mathcal{M}}^{n}\right):=\nu_{p}\left(P_{p, M_{p}^{\uparrow}}^{n}, \boldsymbol{S}_{M_{p}^{\uparrow}}^{n}, C_{p, M_{p}^{\uparrow}}^{n}\right)$ denoting, respectively, the upstream molar fraction and upstream mobility. In (2.22), we have introduced the two-point finite volume approximation of the normal component of the average phase velocity on $\sigma$ given by

$$
F_{p, M, \sigma}\left(\mathcal{X}_{\mathcal{M}}^{n}\right):=|\sigma| \frac{\alpha_{M} \alpha_{L}}{\alpha_{M}+\alpha_{L}}\left[P_{p, M}^{n}-P_{p, L}^{n}+\rho_{p, \sigma}^{n} g\left(z_{M}-z_{L}\right)\right], \quad \alpha_{K}:=\frac{\lambda_{K}}{d_{K \sigma}} \quad \forall K \in\{M, L\},
$$

where $\rho_{p, \sigma}^{n}$ is an approximation of the mass density of the phase $p$ on the face $\sigma$ given by (other choices are possible),

$$
\rho_{p, \sigma}^{n}:=\frac{\left(\chi_{p, M}^{n} \rho_{p}\left(P_{p, M}^{n}, C_{p, M}^{n}\right)+\chi_{p, L}^{n} \rho_{p}\left(P_{p, L}^{n}, C_{p, L}^{n}\right)\right)}{\chi_{p, M}^{n}+\chi_{p, L}^{n}}, \quad \chi_{p, K}^{n}=\left\{\begin{array}{ll}
1 & \text { if } S_{p, K}^{n}>0, \\
0 & \text { otherwise }
\end{array} \quad K \in\{M, L\}\right.
$$

Boundary fluxes are set to zero for all components to account for the homogeneous natural boundary condition 2.6 . 
Remark 2.1 (General meshes and full permeability tensors). A straightforward variation of this scheme that is consistent on more general meshes and for full permeability tensors consists in using a multipoint expression for $F_{p, M, \sigma}(c f$. (2.23) ) in the spirit of [2, 32]; see also [5]. However, resorting to multipoint finite volume methods is only a partial answer to the problem, since those schemes add arithmetic complexity, may suffer from coercivity loss, and are in general nonmonotone.

At the discrete level, volume conservation is expressed by the following relation: For all $1 \leq n \leq N$,

$$
\sum_{p \in \mathcal{P}} S_{p, M}^{n}=1 \quad \forall M \in \mathcal{M}^{n} .
$$

Similarly, the discrete conservation of matter in each phase reads, for all $1 \leq n \leq N$,

$$
\sum_{c \in \mathcal{C}_{p}} C_{p, c, M}^{n}=1 \quad \forall p \in \mathcal{P}, \forall M \in \mathcal{M}^{n} .
$$

Finally, the thermodynamic equilibrium is enforced by requiring the equality of fugacities for all time steps inside each cell, leading to

$$
\sum_{p \in \mathcal{P}} N_{\mathcal{C}_{p}}-N_{\mathcal{C}} \quad \text { equations } \quad \forall 1 \leq n \leq N, \forall M \in \mathcal{M}^{n} .
$$

An important remark which can be exploited in the implementation is that 2.24, 2.25 , and the thermodynamic equilibrium 2.26 express local algebraic relations between the unknowns in each cell. This allows to reduce the size of the global linear system to $N_{\mathcal{M}^{n}} \times N_{\mathcal{C}}$ equations stemming from (2.19). A detailed treatment of local elimination strategies is out of the scope of the present work. We emphasize, however, that a local elimination procedure is indeed used in the numerical examples of Section 5 .

\section{A basic a posteriori error estimate}

We derive here an a posteriori estimate for the error measured by the dual norm of the residual augmented by a nonconformity evaluation term. This choice of the error measure is naturally inspired by the problem, and allows to obtain a fully computable error upper bound. The results of this section are generic for an arbitrary approximation; we show how to apply them to the finite volume setting of Section 2.2 in Section 4 below.

\subsection{Weak solution}

At this stage, we need to characterize a weak solution for the multiphase compositional model 2.1 2.10. Let $(\cdot, \cdot)_{D}$ stand for the $L^{2}$-scalar product on $D \subset \Omega$ and $\|\cdot\|_{D}$ for the associated norm; the same notation is used for both scalar and vector arguments, and the subscript is dropped whenever $D=\Omega$. We define

$$
\begin{aligned}
X & :=L^{2}\left(\left(0, t_{\mathrm{F}}\right) ; H^{1}(\Omega)\right), \\
Y & :=H^{1}\left(\left(0, t_{\mathrm{F}}\right) ; L^{2}(\Omega)\right) .
\end{aligned}
$$

Let $\varepsilon>0$ be a (small) parameter which only needs to satisfy $\varepsilon \leq 1$. We equip the space $X$ with the norm

$$
\|\varphi\|_{X}:=\left\{\sum_{n=1}^{N} \int_{I_{n}} \sum_{M \in \mathcal{M}^{n}}\|\varphi\|_{X, M}^{2} \mathrm{~d} t\right\}^{\frac{1}{2}}, \quad\|\varphi\|_{X, M}^{2}:=\varepsilon h_{M}^{-2}\|\varphi\|_{M}^{2}+\|\nabla \varphi\|_{M}^{2}, \quad \varphi \in X .
$$

This choice is motivated by the homogeneous Neumann boundary condition 2.6); taking $\varepsilon=0$ is possible and classical when Dirichlet (pressure) boundary conditions prescribed at least on a part of the boundary, cf. [35, 68, 20]. In the numerical experiments of Section 5 we set $\varepsilon=1$.

We suppose sufficient regularity to satisfy: 
Assumption 3.1 (Weak solution). There exists a weak solution $\mathcal{X}$ of 2.1 -2.10 which can be characterized as follows:

$$
\begin{aligned}
& l_{c} \in Y \quad \forall c \in \mathcal{C}, \\
& P_{p}(P, \boldsymbol{S}) \in X \quad \forall p \in \mathcal{P}, \\
& \mathbf{\Phi}_{c} \in\left[L^{2}\left(\left(0, t_{\mathrm{F}}\right) ; L^{2}(\Omega)\right)\right]^{d} \quad \forall c \in \mathcal{C}, \\
& \int_{0}^{t_{\mathrm{F}}}\left\{\left(\partial_{t} l_{c}, \varphi\right)(t)-\left(\boldsymbol{\Phi}_{c}, \nabla \varphi\right)(t)\right\} \mathrm{d} t=\int_{0}^{t_{\mathrm{F}}}\left(q_{c}, \varphi\right)(t) \mathrm{d} t \quad \forall \varphi \in X, \forall c \in \mathcal{C}, \\
& \text { the initial condition 2.7 holds, }
\end{aligned}
$$$$
\text { the algebraic closure equations 2.8 }-2.10 \text { hold, }
$$

where $P_{p}, l_{c}$, and $\boldsymbol{\Phi}_{c}$ are defined, respectively, by 2.1), 2.5), and 2.3).

Existence and uniqueness of a weak solution has to our knowledge not been established for the multiphase compositional model. In simplified settings, with typically only two phases present and each phase composed of a single component, such results can be found in [49, 22, 8, 9, 23, 19, 48, 7] and the references therein.

Remark 3.2 (Component fluxes). It follows from (3.3a), the assumption $q_{c} \in L^{2}\left(\left(0, t_{\mathrm{F}}\right) ; L^{2}(\Omega)\right)$, (3.3c), and (3.3d) that actually

$$
\begin{aligned}
\boldsymbol{\Phi}_{c} & \in L^{2}\left(\left(0, t_{\mathrm{F}}\right) ; \mathbf{H}(\operatorname{div}, \Omega)\right) & & \forall c \in \mathcal{C}, \\
\nabla \cdot \boldsymbol{\Phi}_{c} & =q_{c}-\partial_{t} l_{c} & & \forall c \in \mathcal{C}, \\
\boldsymbol{\Phi}_{c} \cdot \boldsymbol{n}_{\Omega} & =0 \quad \text { on } \partial \Omega \times\left(0, t_{\mathrm{F}}\right) & & \forall c \in \mathcal{C},
\end{aligned}
$$

so that the component fluxes $\boldsymbol{\Phi}_{c}$ have the normal trace continuous in a proper sense, the governing equation (2.2) is satisfied with a weak divergence, and the boundary conditions 2.6) hold in the normal trace sense.

\subsection{A generic approximate solution}

In order to present the results of this section abstractly, not linked to any specific numerical discretization, we suppose here that for each $0 \leq n \leq N$ and $p \in \mathcal{P}$, we are given a piecewise $H^{1}$ in space (typically piecewise polynomial of degree $\geq 1$, possibly discontinuous) phase pressure $P_{p, h}^{n}$. Therefrom, the spacetime functions $P_{p, h \tau}$ are created by prescribing $P_{p, h \tau}\left(t^{n}\right):=P_{p, h}^{n}, 0 \leq n \leq N, P_{p, h \tau}$ thus being piecewise affine and continuous in time. By such an assumption, $P_{p, h \tau}$ are not necessarily included in the energy space $X$; we henceforth understand by $\nabla$ the broken gradient operator on the meshes $\mathcal{M}^{n}$. Similarly, the amounts of components $l_{c, h}^{n}, 0 \leq n \leq N, c \in \mathcal{C}$, are supposed $L^{2}$ in space (typically piecewise polynomial of degree $\geq 0$, possibly discontinuous) and form the piecewise affine and continuous-in-time functions $l_{c, h \tau}, c \in \mathcal{C}$, by $l_{c, h \tau}\left(t^{n}\right)=l_{c, h}^{n}$. Thus $l_{c, h \tau} \in Y$, in a discrete equivalent of (3.3a).

We suppose that the space-time reference pressure, saturation, and molar fraction approximations $P_{h \tau}, S_{p, h \tau}$, and $C_{p, c, h \tau}, p \in \mathcal{P}, c \in \mathcal{C}_{p}$ are linked to $P_{p, h \tau}$ and $l_{c, h \tau}$ via 2.1 and 2.5, respectively. Similarly, we suppose that the algebraic closure equations (2.8)-2.10) are satisfied exactly, and, for simplicity, that $l_{c, h}^{0}$ satisfies exactly the initial condition 2.7 , i.e., $l_{c, h}^{0}=l_{c}^{0}$. Below, the concise notation for the vector-valued space-time functions $\boldsymbol{P}_{h \tau}:=\left(P_{p, h \tau}\right)_{p \in \mathcal{P}}, \boldsymbol{S}_{h \tau}:=\left(S_{p, h \tau}\right)_{p \in \mathcal{P}}$ and, for all $p \in \mathcal{P}$, $C_{p, h \tau}:=\left(C_{p, c, h \tau}\right)_{c \in \mathcal{C}_{p}}$, will be employed. We show how we obtain the above quantities in the finite volume setting of Section 2.2 , or more precisely during the calculation including also an iterative linearization and iterative solution of the arising linear systems, in Section 4.2 below.

\subsection{Error measure}

Following [68, we consider an error measure for the above approximate solution inspired from the weak formulation (3.3), which consists of the dual norm of the residual supplemented by a nonconformity evaluation term. For nonlinear problems, it has been argued in, e.g., [21, 33, 36, 29, that the dual norm of the residual is a more natural choice than the energy norm. Moreover, in the two-phase flow setting with conforming approximations and Dirichlet boundary conditions, it has been shown in [20, 
Theorem 5.7] that the dual norm of the residual is an upper bound for an energy-type difference between the exact pressures and saturations. Concretely, our error measure is defined as

$$
\mathcal{N}=\mathcal{N}\left(\boldsymbol{P}_{h \tau}, \boldsymbol{S}_{h \tau},\left(\boldsymbol{C}_{p, h \tau}\right)_{p \in \mathcal{P}}\right):=\left\{\sum_{c \in \mathcal{C}} \mathcal{N}_{c}^{2}\right\}^{\frac{1}{2}}+\left\{\sum_{p \in \mathcal{P}} \mathcal{N}_{p}^{2}\right\}^{\frac{1}{2}}
$$

where the quantities $\mathcal{N}_{c}, c \in \mathcal{C}$, and $\mathcal{N}_{p}, p \in \mathcal{P}$, have the same dependence as $\mathcal{N}$. They are defined, respectively, as

$$
\mathcal{N}_{c}:=\sup _{\varphi \in X,\|\varphi\|_{X}=1} \int_{0}^{t_{\mathrm{F}}}\left\{\left(\partial_{t} l_{c}-\partial_{t} l_{c, h \tau}, \varphi\right)(t)-\left(\mathbf{\Phi}_{c}-\boldsymbol{\Phi}_{c, h \tau}, \nabla \varphi\right)(t)\right\} \mathrm{d} t,
$$

with the exact component fluxes $\boldsymbol{\Phi}_{c}$ defined by 2.3 and $\boldsymbol{\Phi}_{c, h \tau}$ given by

$$
\boldsymbol{\Phi}_{c, h \tau}:=\sum_{p \in \mathcal{P}_{c}} \boldsymbol{\Phi}_{p, c, h \tau}, \quad \boldsymbol{\Phi}_{p, c, h \tau}:=\nu_{p}\left(P_{p, h \tau}, \boldsymbol{S}_{h \tau}, \boldsymbol{C}_{p, h \tau}\right) C_{p, c, h \tau} \boldsymbol{v}_{p}\left(P_{p, h \tau}, \boldsymbol{C}_{p, h \tau}\right),
$$

and

$$
\mathcal{N}_{p}:=\inf _{\delta_{p} \in X}\left\{\sum_{c \in \mathcal{C}_{p}} \int_{0}^{t_{\mathrm{F}}}\left\|\boldsymbol{\Psi}_{p, c}\left(P_{p, h \tau}\right)(t)-\boldsymbol{\Psi}_{p, c}\left(\delta_{p}\right)(t)\right\|^{2} \mathrm{~d} t\right\}^{\frac{1}{2}},
$$

where, for a space-time function $\varphi \in L^{2}\left(\left(0, t_{\mathrm{F}}\right) ; H^{1}(\mathcal{M})\right)$ (piecewise regular with respect to the partitions $\mathcal{M}^{n}$ ), we have let

$$
\boldsymbol{\Psi}_{p, c}(\varphi):=\nu_{p}\left(P_{p, h \tau}, \boldsymbol{S}_{h \tau}, \boldsymbol{C}_{p, h \tau}\right) C_{p, c, h \tau} \boldsymbol{\Lambda} \nabla \varphi .
$$

The first term $\mathcal{N}_{c}$ evaluates the non-satisfaction of (3.3d) at the discrete level, as $\boldsymbol{\Phi}_{c, h \tau}$ given by (3.7) do not necessarily satisfy the conditions (3.4), while the second term $\mathcal{N}_{p}$ quantifies the possible departure of the discrete phase pressures $P_{p, h \tau}$ from the energy space $X$.

\subsection{Flux and pressure reconstructions}

To estimate the terms $\mathcal{N}_{c}$ in the error measure (3.5) we, for all $1 \leq n \leq N$, introduce $N_{\mathcal{C}}$ component flux reconstructions $\left(\boldsymbol{\Theta}_{c, h}^{n}\right)_{c \in \mathcal{C}}$ such that, for all $c \in \mathcal{C}, \boldsymbol{\Theta}_{c, h}^{n} \in \mathbf{H}(\operatorname{div} ; \Omega)$ and the following local conservation property holds:

$$
\left(q_{c, h}^{n}-\partial_{t}^{n} l_{c, h \tau}-\nabla \cdot \Theta_{c, h}^{n}, 1\right)_{M}=0 \quad \forall c \in \mathcal{C}, \forall M \in \mathcal{M}^{n},
$$

where we have introduced the piecewise constant space functions $q_{c, h}^{n}, c \in \mathcal{C}$, such that $\left.\left(q_{c, h}^{n}\right)\right|_{M}=$ $\int_{I_{n}} \int_{M} q_{c} /\left(|M| \tau_{n}\right) \mathrm{d} \mathbf{x} \mathrm{d} t$. For further use we also define the space-time functions $q_{c, h \tau}, c \in \mathcal{C}$, such that $\left.q_{c, h \tau}\right|_{I_{n}}=q_{c, h}^{n}$ for all $1 \leq n \leq N$. It is also assumed that the boundary condition (2.6) is satisfied exactly, i.e.,

$$
\mathbf{\Theta}_{c, h}^{n} \cdot \boldsymbol{n}_{\Omega}=0 \quad \text { on } \partial \Omega .
$$

We denote by $\boldsymbol{\Theta}_{c, h \tau}$ the space-time function such that $\boldsymbol{\Theta}_{c, h \tau \mid I_{n}}=\boldsymbol{\Theta}_{c, h}^{n}$ for all $1 \leq n \leq N$. Note that $\boldsymbol{\Theta}_{c, h \tau}$ mimic the properties of the weak component fluxes $\boldsymbol{\Phi}_{c}$ as expressed in Remark 3.2. In practice, $\boldsymbol{\Theta}_{c, h}^{n}$ are constructed in the Raviart-Thomas-Nédélec finite-dimensional subspaces of $\mathbf{H}($ div; $\Omega$ ); details in the finite volume context are given in Section 4.4 below.

To estimate the terms $\mathcal{N}_{p}$ in (3.5), we need $N_{\mathcal{P}}$ phase pressure reconstructions $\mathfrak{P}_{p, h \tau}, p \in \mathcal{P}$, such that $\mathfrak{P}_{p, h \tau} \in X$ for all $p \in \mathcal{P}$. These reconstructions are typically piecewise polynomial continuous in space and piecewise affine continuous in time. Details in the finite volume context are given in Section 4.3 below.

\subsection{A posteriori error estimate}

We now derive a fully computable upper bound for the approximate solution as specified in Section 3.2 . the error measure introduced in Section 3.3 and based on the pressure and flux reconstructions of Section 3.4. A key ingredient is the following Poincaré inequality:

$$
\left\|\varphi-\varphi_{M}\right\|_{M} \leq C_{\mathrm{P}, M} h_{M}\|\nabla \varphi\|_{M} \quad \forall \varphi \in H^{1}(M), \forall M \in \mathcal{M}^{n}, 1 \leq n \leq N,
$$


where $\varphi_{M}:=\int_{M} \varphi /|M| \mathrm{d} \mathbf{x}$ denotes the mean value of the function $\varphi$ on $M$. We recall that $C_{\mathrm{P}, M}=1 / \pi$ for convex cells $M$ (see [60, 15]).

For all $1 \leq n \leq N, M \in \mathcal{M}^{n}$, and $c \in \mathcal{C}$, we define the residual estimators $\eta_{\mathrm{R}, M, c}^{n}$, the flux estimators $\eta_{\mathrm{F}, M, c}^{n}(t), t \in I_{n}$, and the nonconformity estimators $\eta_{\mathrm{NC}, M, p, c}^{n}(t), t \in I_{n}, p \in \mathcal{P}_{c}$, as follows:

$$
\begin{aligned}
\eta_{\mathrm{R}, M, c}^{n} & :=\min \left\{C_{\mathrm{P}, M}, \varepsilon^{-\frac{1}{2}}\right\} h_{M}\left\|q_{c, h}^{n}-\partial_{t}^{n} l_{c, h \tau}-\nabla \cdot \boldsymbol{\Theta}_{c, h}^{n}\right\|_{M}, \\
\eta_{\mathrm{F}, M, c}^{n}(t) & :=\left\|\boldsymbol{\Theta}_{c, h}^{n}-\mathbf{\Phi}_{c, h \tau}(t)\right\|_{M}, \\
\eta_{\mathrm{NC}, M, p, c}^{n}(t) & :=\left\|\mathbf{\Psi}_{p, c}\left(P_{p, h \tau}\right)(t)-\mathbf{\Psi}_{p, c}\left(\mathfrak{P}_{p, h \tau}\right)(t)\right\|_{M} .
\end{aligned}
$$

Theorem 3.3 (A posteriori estimate for the error measure (3.5)). Under Assumption 3.1, for the flux and pressure reconstructions of Section 3.4, and with the estimators given by (3.12), there holds

$$
\begin{aligned}
\mathcal{N}_{c} & \leq\left\{\sum_{n=1}^{N} \int_{I_{n}} \sum_{M \in \mathcal{M}^{n}}\left(\eta_{\mathrm{R}, M, c}^{n}+\eta_{\mathrm{F}, M, c}^{n}(t)\right)^{2} \mathrm{~d} t\right\}^{\frac{1}{2}}+\left\|q_{c}-q_{c, h \tau}\right\|_{X^{\prime}} \quad c \in \mathcal{C}, \\
\mathcal{N}_{p} & \leq\left\{\sum_{c \in \mathcal{C}_{p}} \sum_{n=1}^{N} \int_{I_{n}} \sum_{M \in \mathcal{M}^{n}}\left(\eta_{\mathrm{NC}, M, p, c}^{n}(t)\right)^{2} \mathrm{~d} t\right\}^{\frac{1}{2}} \quad p \in \mathcal{P} .
\end{aligned}
$$

Remark 3.4 (Source term). In reservoir simulation, the source terms $q_{c}, c \in \mathcal{C}$, are typically piecewise constant on the space-time mesh. Then, the last term in the estimate (3.13a), called data oscillation in numerical analysis literature, vanishes.

Proof. The proof is simple using the equilibrated flux reconstructions $\boldsymbol{\Theta}_{c, h}^{n}$. To bound $\mathcal{N}_{c}$, let $\varphi \in X$ be such that $\|\varphi\|_{X}=1$. There holds

$$
\begin{aligned}
\Gamma(\varphi):= & \int_{0}^{t_{\mathrm{F}}}\left\{\left(\partial_{t} l_{c}-\partial_{t} l_{c, h \tau}, \varphi\right)(t)-\left(\mathbf{\Phi}_{c}-\boldsymbol{\Phi}_{c, h \tau}, \nabla \varphi\right)(t)\right\} \mathrm{d} t \\
= & \int_{0}^{t_{\mathrm{F}}}\left\{\left(q_{c}-\partial_{t} l_{c, h \tau}, \varphi\right)(t)+\left(\mathbf{\Phi}_{c, h \tau}, \nabla \varphi\right)(t)\right\} \mathrm{d} t \\
= & \int_{0}^{t_{\mathrm{F}}}\left\{\left(q_{c, h \tau}-\partial_{t} l_{c, h \tau}-\nabla \cdot \boldsymbol{\Theta}_{c, h \tau}, \varphi\right)(t)-\left(\boldsymbol{\Theta}_{c, h \tau}-\boldsymbol{\Phi}_{c, h \tau}, \nabla \varphi\right)(t)\right\} \mathrm{d} t \\
& \quad+\int_{0}^{t_{\mathrm{F}}}\left(q_{c}-q_{c, h \tau}, \varphi\right)(t) \mathrm{d} t \\
= & \sum_{n=1}^{N} \int_{I_{n}}\left\{\left(q_{c, h}^{n}-\partial_{t}^{n} l_{c, h \tau}-\nabla \cdot \boldsymbol{\Theta}_{c, h}^{n}, \varphi\right)(t)-\left(\Theta_{c, h}^{n}-\mathbf{\Phi}_{c, h \tau}, \nabla \varphi\right)(t)\right\} \mathrm{d} t \\
& \quad+\int_{0}^{t_{\mathrm{F}}}\left(q_{c}-q_{c, h \tau}, \varphi\right)(t) \mathrm{d} t,
\end{aligned}
$$

where we have used $(3.3 \mathrm{~d})$ in the second line and where we have added and subtracted $\left(\boldsymbol{\Theta}_{c, h \tau}, \nabla \varphi\right)(t)$ and used Green's theorem along with (3.10b) to infer $\left(\nabla \cdot \boldsymbol{\Theta}_{c, h \tau}, \varphi\right)(t)+\left(\boldsymbol{\Theta}_{c, h \tau}, \nabla \varphi\right)(t)=0$ in the third line. For all $1 \leq n \leq N$ and $t \in I_{n}$, using the local conservation property (3.10a) followed by the Cauchy-Schwarz and Poincaré's (3.11) inequalities, and recalling (3.12a), it is inferred,

$$
\begin{aligned}
\left(q_{c, h}^{n}-\partial_{t}^{n} l_{c, h \tau}-\nabla \cdot \Theta_{c, h}^{n}, \varphi\right)(t) & =\sum_{M \in \mathcal{M}^{n}}\left(q_{c, h}^{n}-\partial_{t}^{n} l_{c, h \tau}-\nabla \cdot \Theta_{c, h}^{n}, \varphi\right)_{M}(t) \\
& =\sum_{M \in \mathcal{M}^{n}}\left(q_{c, h}^{n}-\partial_{t}^{n} l_{c, h \tau}-\nabla \cdot \Theta_{c, h}^{n}, \varphi-\varphi_{M}\right)_{M}(t) \\
& \leq \sum_{M \in \mathcal{M}^{n}}\left\|q_{c, h}^{n}-\partial_{t}^{n} l_{c, h \tau}-\nabla \cdot \Theta_{c, h}^{n}\right\|_{M}\left\|\varphi-\varphi_{M}\right\|_{M}(t) \\
& \leq \sum_{M \in \mathcal{M}^{n}} \min \left\{C_{\mathrm{P}, M}, \varepsilon^{-\frac{1}{2}}\right\} h_{M}\left\|q_{c, h}^{n}-\partial_{t}^{n} l_{c, h \tau}-\nabla \cdot \Theta_{c, h}^{n}\right\|_{M}\|\varphi\|_{X, M}(t) \\
& =\sum_{M \in \mathcal{M}^{n}} \eta_{\mathrm{R}, M, c}^{n}\|\varphi\|_{X, M}(t) .
\end{aligned}
$$


Using again the Cauchy-Schwarz inequality yields for all $1 \leq n \leq N$ and $t \in I_{n}$,

$$
-\left(\boldsymbol{\Theta}_{c, h}^{n}-\boldsymbol{\Phi}_{c, h \tau}, \nabla \varphi\right)(t)=\sum_{M \in \mathcal{M}^{n}}-\left(\Theta_{c, h}^{n}-\boldsymbol{\Phi}_{c, h \tau}, \nabla \varphi\right)_{M}(t) \leq \sum_{M \in \mathcal{M}^{n}} \eta_{\mathrm{F}, M, c}^{n}(t)\|\nabla \varphi\|_{M}(t) .
$$

Thus,

$$
\Gamma(\varphi) \leq \sum_{n=1}^{N} \int_{I_{n}} \sum_{M \in \mathcal{M}^{n}}\left\{\left(\eta_{\mathrm{R}, M, c}^{n}+\eta_{\mathrm{F}, M, c}^{n}(t)\right)\|\varphi\|_{X, M}(t)\right\} \mathrm{d} t+\left\|q_{c}-q_{c, h \tau}\right\|_{X^{\prime}}\|\varphi\|_{X} .
$$

Finally, using the Cauchy-Schwarz inequality together with the definition 3.2 of the norm on $X$ and $\|\varphi\|_{X}=1$ to bound the first term in equation (3.14) yields the estimate (3.13a).

The estimate (3.13b) is obtained using the $X$-regularity of the phase pressure reconstructions $\mathfrak{P}_{p, h \tau}$ defined in Section 3.4 to bound the infimum in 3.8 .

\section{Application to finite volume method and adaptivity based on distinguishing the different error components}

We apply here the abstract result of the previous section to the finite volume discretization introduced in Section 2.2. Moreover, we consider a practical implementation of (2.17)- 2.26 , requiring the solution of the arising system of nonlinear algebraic equations at each time step. Distinguishing the different error components in the basic a posteriori error estimate of Theorem 3.3, we propose stopping criteria for the employed iterative algebraic and linearization solvers. An entirely adaptive algorithm, also balancing the time and space error components via adaptive time step choice and adaptive mesh refinement, is proposed. For the sake of simplicity, we assume henceforth that the source terms $q_{c}, c \in \mathcal{C}$, are piecewise constant on the space-time mesh, so that the last term in the estimate 3.13a vanishes, cf. Remark 3.4

\subsection{Linearization and algebraic resolution}

The finite volume method discussed in Section 2.2 requires to solve a system of nonlinear algebraic equations at each time step. Recalling (2.16), for all $1 \leq n \leq N$, the discrete conservation of components 2.19$)$ can be rewritten as

$$
R_{c, M}^{n}\left(\mathcal{X}_{\mathcal{M}}^{n}\right):=\frac{|M|}{\tau^{n}}\left(l_{c, M}\left(\mathcal{X}_{M}^{n}\right)-l_{c, M}^{n-1}\right)+\sum_{\sigma \in \mathcal{E}_{M}^{\mathrm{i}, n}} F_{c, M, \sigma}\left(\mathcal{X}_{\mathcal{M}}^{n}\right)-|M| q_{c, M}^{n}=0 \quad \forall c \in \mathcal{C}, \forall M \in \mathcal{M}^{n}
$$

System 4.1 can be solved by any suitable linearization. In what follows, we focus on the Newton linearization algorithm, although the a posteriori error analysis developed in this work can be easily adapted to accommodate other linearization algorithms in the spirit of 36 .

For $1 \leq n \leq N$ and $\mathcal{X}_{\mathcal{M}}^{n, 0}$ fixed (typically, $\mathcal{X}_{\mathcal{M}}^{n, 0}=\mathcal{X}_{\mathcal{M}}^{n-1}$ ), the Newton algorithm generates a sequence $\left(\mathcal{X}_{\mathcal{M}}^{n, k}\right)_{k \geq 1}$ with $\mathcal{X}_{\mathcal{M}}^{n, k}$ solution to the following system of linear algebraic equations: For all $c \in \mathcal{C}$ and all $M \in \mathcal{M}^{n}$,

$$
\sum_{M^{\prime} \in \mathcal{M}^{n}} \frac{\partial R_{c, M}^{n}}{\partial \mathcal{X}_{M^{\prime}}^{n}}\left(\mathcal{X}_{\mathcal{M}}^{n, k-1}\right) \cdot\left(\mathcal{X}_{M^{\prime}}^{n, k}-\mathcal{X}_{M^{\prime}}^{n, k-1}\right)+R_{c, M}^{n}\left(\mathcal{X}_{\mathcal{M}}^{n, k-1}\right)=0
$$

The (approximate) solution to 4.2 is typically obtained using an iterative algebraic solver. For $1 \leq n \leq$ $N$, a given Newton iteration $k \geq 1$, and $\mathcal{X}_{\mathcal{M}}^{n, k, 0}$ fixed (typically, $\mathcal{X}_{\mathcal{M}}^{n, k, 0}=\mathcal{X}_{\mathcal{M}}^{n, k-1}$ ), the iterative solver generates a sequence $\left(\mathcal{X}_{\mathcal{M}}^{n, k, i}\right)_{i \geq 1}$ solving 4.2 up to the residuals, given for all $c \in \mathcal{C}$ and all $M \in \mathcal{M}^{n}$ by

$$
R_{c, M}^{n, k, i}:=\sum_{M^{\prime} \in \mathcal{M}^{n}} \frac{\partial R_{c, M}^{n}}{\partial \mathcal{X}_{M^{\prime}}^{n}}\left(\mathcal{X}_{\mathcal{M}}^{n, k-1}\right) \cdot\left(\mathcal{X}_{M^{\prime}}^{n, k, i}-\mathcal{X}_{M^{\prime}}^{n, k-1}\right)+R_{c, M}^{n}\left(\mathcal{X}_{\mathcal{M}}^{n, k-1}\right)
$$

Plugging 4.1] into 4.3), it is inferred

$$
\begin{aligned}
R_{c, M}^{n, k, i}= & \sum_{M^{\prime} \in \mathcal{M}^{n}} \frac{|M|}{\tau^{n}} \frac{\partial l_{c, M}}{\partial \mathcal{X}_{M^{\prime}}^{n}}\left(\mathcal{X}_{\mathcal{M}}^{n, k-1}\right) \cdot\left(\mathcal{X}_{M^{\prime}}^{n, k, i}-\mathcal{X}_{M^{\prime}}^{n, k-1}\right) \\
& +\sum_{M^{\prime} \in \mathcal{M}^{n}} \sum_{\sigma \in \mathcal{E}_{M}^{\mathrm{i}, n}} \frac{\partial F_{c, M, \sigma}}{\partial \mathcal{X}_{M^{\prime}}^{n}}\left(\mathcal{X}_{\mathcal{M}}^{n, k-1}\right) \cdot\left(\mathcal{X}_{M^{\prime}}^{n, k, i}-\mathcal{X}_{M^{\prime}}^{n, k-1}\right)+R_{c, M}^{n}\left(\mathcal{X}_{\mathcal{M}}^{n, k-1}\right) .
\end{aligned}
$$


The first and the second terms in the right-hand side of (4.4) are linear perturbations of the corresponding terms in 4.1. The linear perturbation in the accumulation is

$$
\mathcal{L}_{c, M}^{n, k, i}:=\sum_{M^{\prime} \in \mathcal{M}^{n}} \frac{\partial l_{c, M}}{\partial \mathcal{X}_{M^{\prime}}^{n}}\left(\mathcal{X}_{\mathcal{M}}^{n, k-1}\right) \cdot\left(\mathcal{X}_{M^{\prime}}^{n, k, i}-\mathcal{X}_{M^{\prime}}^{n, k-1}\right)
$$

whereas the linearized component flux reads

$$
F_{c, M, \sigma}^{n, k, i}:=\sum_{p \in \mathcal{P}_{c}} F_{p, c, M, \sigma}^{n, k, i},
$$

with linearized phase component fluxes

$$
F_{p, c, M, \sigma}^{n, k, i}:=F_{p, c, M, \sigma}\left(\mathcal{X}_{\mathcal{M}}^{n, k-1}\right)+\sum_{M^{\prime} \in \mathcal{M}^{n}} \frac{\partial F_{p, c, M, \sigma}}{\partial \mathcal{X}_{M^{\prime}}^{n}}\left(\mathcal{X}_{\mathcal{M}}^{n, k-1}\right) \cdot\left(\mathcal{X}_{M^{\prime}}^{n, k, i}-\mathcal{X}_{M^{\prime}}^{n, k-1}\right) .
$$

In conclusion, at time step $n$, Newton iteration $k \geq 1$, and linear solver iteration $i \geq 1$, the residual vector $R_{c, M}^{n, k, i}$ is given by

$$
R_{c, M}^{n, k, i}=\frac{|M|}{\tau^{n}}\left(l_{c, M}\left(\mathcal{X}_{\mathcal{M}}^{n, k-1}\right)+\mathcal{L}_{c, M}^{n, k, i}-l_{c, M}^{n-1}\right)+\sum_{\sigma \in \mathcal{E}_{M}^{\mathrm{i}, n}} F_{c, M, \sigma}^{n, k, i}-|M| q_{c, M}^{n} \quad \forall c \in \mathcal{C}, \forall M \in \mathcal{M}^{n}
$$

\subsection{Approximate solution}

In this section we identify the approximate solutions, as discussed in Section 3.2 , for the finite volume setting of Sections 2.2 and 4.1. We will need some finite-dimensional subspaces of $\mathbf{H}(\operatorname{div}, \Omega)$. When the meshes $\mathcal{M}^{n}$ consist of rectangular parallelepipeds, as it is the case in the numerical experiments of Section 5 below, we use the lowest-order Raviart-Thomas-Nédélec spaces

$$
\begin{aligned}
\boldsymbol{R T N}\left(\mathcal{M}^{n}\right):= & \left\{\boldsymbol{v}_{h} \in \mathbf{H}(\operatorname{div} ; \Omega) ;\left.\boldsymbol{v}_{h}\right|_{M} \in \mathbb{Q}_{0,1}(M) \times \mathbb{Q}_{1,0}(M) \text { if } d=2,\right. \\
& \left.\mathbb{Q}_{0,1,1}(M) \times \mathbb{Q}_{1,0,1}(M) \times \mathbb{Q}_{1,1,0}(M) \text { if } d=3, \forall M \in \mathcal{M}^{n}\right\},
\end{aligned}
$$

whereas for simplicial meshes, we employ

$$
\boldsymbol{R T N}\left(\mathcal{M}^{n}\right):=\left\{\boldsymbol{v}_{h} \in \mathbf{H}(\operatorname{div} ; \Omega) ;\left.\boldsymbol{v}_{h}\right|_{M} \in\left[\mathbb{P}_{0}(M)\right]^{d}+\boldsymbol{x} \mathbb{P}_{0}(M), \forall M \in \mathcal{M}^{n}\right\},
$$

see Brezzi and Fortin [17] for details.

Remark 4.1 (General meshes). For general polygonal meshes, one option is to introduce matching simplicial submeshes of $\mathcal{M}^{n}$ and use 4.10. One can alternatively consider the constructions proposed in [52, [51], [27], or [69].

\subsubsection{Phase pressure postprocessings}

As explained in Sections 3.2 and 3.3 , we need to evaluate the broken gradient of the discrete phase pressures $P_{p, h}^{n}, p \in \mathcal{P}, 0 \leq n \leq N$. The original finite volume pressure approximations $P_{p, M}^{n}$ of 2.18 , or, more precisely, $P_{p, M}^{n, k, i}$ obtained from $\mathcal{X}_{\mathcal{M}}^{n, k, i}$ in Section 4.1. are only piecewise constant. We thus, following [67, define piecewise quadratic, possibly discontinuous phase pressures as follows. Let $1 \leq n \leq$ $N$, a Newton linearization iteration $k \geq 1$, and an algebraic solver iteration $i \geq 1$ be fixed. For all $p \in \mathcal{P}$ we define $\boldsymbol{\Gamma}_{p, h}^{n, k, i} \in \mathbf{R T N}\left(\mathcal{M}^{n}\right)$ such that, for all $M \in \mathcal{M}^{n}$ and all $\sigma \in \mathcal{E}_{M}^{\mathrm{i}, n}$,

$$
\left(\boldsymbol{\Gamma}_{p, h}^{n, k, i} \cdot \boldsymbol{n}_{M}, 1\right)_{\sigma}=F_{p, M, \sigma}\left(\mathcal{X}_{\mathcal{M}}^{n, k, i}\right),
$$

with $F_{p, M, \sigma}$ defined by 2.23 and $\boldsymbol{\Gamma}_{p, h}^{n, k, i} \cdot \boldsymbol{n}_{\Omega}=0$ on $\partial \Omega$. The fluxes $\boldsymbol{\Gamma}_{p, h}^{n, k, i}$ are thus discrete versions of the Darcy velocities $\boldsymbol{v}_{p}$ from (2.4). Motivated by (2.4), we then, for each $p \in \mathcal{P}$, introduce the piecewise quadratic phase pressure $P_{p, h}^{n, k, i}$ such that, for all $M \in \mathcal{M}^{n}$,

$$
\left.\left(-\boldsymbol{\Lambda} \nabla P_{p, h}^{n, k, i}\right)\right|_{M}=\left.\left(\boldsymbol{\Gamma}_{p, h}^{n, k, i}\right)\right|_{M}-\left.\left(\boldsymbol{\Lambda} \rho_{p}\left(P_{p, M}^{n, k, i}, \boldsymbol{C}_{p, M}^{n, k, i}\right) \boldsymbol{g}\right)\right|_{M} \quad \text { and } \quad \frac{\left(P_{p, h}^{n, k, i}, 1\right)_{M}}{|M|}=P_{p, M}^{n, k, i}
$$

The space-time function $P_{p, h \tau}^{n, k, i}$ is then as usual continuous and piecewise affine in time, given by $P_{p, h}^{n, k, i}$ at the discrete times $t^{n}$; for $n=0$, the initial datum from 2.17) is used. 
Remark 4.2 (General meshes). On general polygonal or polyhedral meshes, one can either again introduce a simplicial submesh, or define for all $p \in \mathcal{P}$ a piecewise affine, possibly discontinuous pressure $P_{p, h}^{n, k, i}$, replacing the Raviart-Thomas-Nédélec vectors $\left.\left(\boldsymbol{\Gamma}_{p, h}^{n, k, i}\right)\right|_{M}$ in 4.11 by the constant vectors

$$
\sum_{\sigma \in \mathcal{E}_{M}^{\mathrm{i}, n}} \frac{|\sigma|}{|M|} F_{p, M, \sigma}\left(\mathcal{X}_{\mathcal{M}}^{n, k, i}\right)\left(\boldsymbol{x}_{\sigma}-\boldsymbol{x}_{M}\right)
$$

The use of the above formula to lift fluxes is justified in [5, Section 2.3].

\subsubsection{Reference pressure, saturations, molar fractions, and amounts of components}

The approximations of all saturations, molar fractions, and amounts of components by the finite volume approach of Sections 2.2 and 4.1 is piecewise constant on the meshes $\mathcal{M}^{n}$. We keep them as such and use the notations (recall the definition of the function $l_{c, M}$ of 2.20$)$ )

$$
\begin{aligned}
\left.\left(S_{p, h}^{n, k, i}\right)\right|_{M} & =S_{p, M}^{n, k, i} \\
\left.\left(C_{p, c, h}^{n, k, i}\right)\right|_{M} & =C_{p, c, M}^{n, k, i}, \\
\left.\left(l_{c, h}^{n, k, i}\right)\right|_{M} & =l_{c, M}^{n, k, i}:=l_{c, M}\left(\mathcal{X}_{\mathcal{M}}^{n, k, i}\right),
\end{aligned}
$$

for $0 \leq n \leq N, k \geq 1, i \geq 1, M \in \mathcal{M}^{n}, p \in \mathcal{P}$, and $c \in \mathcal{C}_{p}$. The space-time functions $S_{p, h \tau}^{n, k, i}, C_{p, c, h \tau}^{n, k, i}$, and $l_{c, h \tau}^{n, k, i}$ are then defined therefrom while being continuous and piecewise affine in time. In what concerns the reference pressure $P_{h \tau}^{n, k, i}$, it does not appear explicitly in what follows.

In Section 3.2 we have made the assumption that the links (2.1) and 2.5), as well as the algebraic closure equations 2.8 -2.10, are satisfied exactly for the discrete approximations $P_{h \tau}^{n, k, i}, S_{p, h \tau}^{n, k, i}, C_{p, c, h \tau}^{n, k, i}$, $P_{p, h \tau}^{n, k, i}$, and $l_{c, h \tau}^{n, k, i}$. This may not hold precisely for all of the required links for the above construction but we suppose the error from this non-satisfaction negligible. Typically $(2.8)$ and $\sqrt{2.9}$ hold precisely but (2.1) and 2.5 are violated (the capillary pressure function applied to a piecewise polynomial is typically no more a piecewise polynomial and a product of two piecewise affine-in-time functions is a piecewise quadratic-in-time function) and (2.10) will be violated if the local fugacity equations are not resolved exactly.

\subsection{Phase pressure reconstructions}

We define the phase pressure reconstructions discussed in Section $\left(3.4\right.$ from $P_{p, h}^{n, k, i}$ of 4.11 by

$$
\mathfrak{P}_{p, h}^{n, k, i}=\mathcal{I}\left(P_{p, h}^{n, k, i}\right) \quad p \in \mathcal{P}
$$

$0 \leq n \leq N, k \geq 1, i \geq 1$, where $\mathcal{I}$ denotes the vertex-averaging interpolator. This operator has been introduced in the context of a posteriori error estimates for finite volume discretizations of Darcy's equations by Achdou et al. [3] and in the discontinuous Galerkin setting by Karakashian and Pascal [47. As usual, $\mathfrak{P}_{p, h \tau}$ is then continuous and piecewise affine in time, given by $\mathfrak{P}_{p, h}^{n, k, i}$ at the discrete times $t^{n}$. Most importantly, it satisfies $\mathfrak{P}_{p, h \tau} \in X$.

\subsection{Component flux reconstructions}

We provide here details on how to build the component flux reconstructions in the spirit of Section 3.4 for the finite volume setting of Sections 2.2 and 4.1 . Several different flux reconstructions will be introduced to accommodate the presence of different error components.

Let a time step $1 \leq n \leq N$, a Newton linearization iteration $k \geq 1$, and an algebraic solver iteration $i \geq 1$ be fixed. For all $c \in \mathcal{C}$, the discretization flux reconstruction $\boldsymbol{\Theta}_{\mathrm{disc}, c, h}^{n, k, i} \in \mathbf{R T N}\left(\mathcal{M}^{n}\right)$ is such that, for all $M \in \mathcal{M}^{n}$ and all $\sigma \in \mathcal{E}_{M}^{\mathrm{i}, n}$,

$$
\left(\Theta_{\mathrm{disc}, c, h}^{n, k, i} \cdot \boldsymbol{n}_{M}, 1\right)_{\sigma}:=F_{c, M, \sigma}\left(\mathcal{X}_{\mathcal{M}}^{n, k, i}\right),
$$


with $F_{c, M, \sigma}$ defined by 2.21), while $\boldsymbol{\Theta}_{\text {disc }, c, h}^{n, k, i} \cdot \boldsymbol{n}_{\Omega}=0$ on $\partial \Omega$ coherently with 2.6). For all $c \in \mathcal{C}$ we also define a linearization error flux reconstruction $\Theta_{\operatorname{lin}, c, h}^{n, k, i} \in \mathbf{R T N}\left(\mathcal{M}^{n}\right)$ such that, for all $M \in \mathcal{M}^{n}$ and for all $\sigma \in \mathcal{E}_{M}^{\mathrm{i}, n}$,

$$
\left(\boldsymbol{\Theta}_{\operatorname{lin}, c, h}^{n, k, i} \cdot \boldsymbol{n}_{M}, 1\right)_{\sigma}=F_{c, M, \sigma}^{n, k, i}-F_{c, M, \sigma}\left(\mathcal{X}_{\mathcal{M}}^{n, k, i}\right),
$$

with $F_{c, M, \sigma}^{n, k, i}$ defined by (4.6), and, similarly, an algebraic error flux reconstruction $\Theta_{\mathrm{alg}, c, h}^{n, k, i} \in \mathbf{R T N}\left(\mathcal{M}^{n}\right)$ such that, for all $M \in \mathcal{M}^{n}$ and for all $\sigma \in \mathcal{E}_{M}^{\mathrm{i}, n}$,

$$
\left(\Theta_{\mathrm{alg}, c, h}^{n, k, i} \cdot \boldsymbol{n}_{M}, 1\right)_{\partial M}:=-R_{c, M}^{n, k, i},
$$

with $R_{c, M}^{n, k, i}$ defined by 4.8. To complete both 4.14b and 4.14c), we set respectively $\Theta_{\operatorname{lin}, c, h}^{n, k, i} \cdot \boldsymbol{n}_{\Omega}=0$ and $\boldsymbol{\Theta}_{\text {als,c.h }}^{n, k, i} \cdot \boldsymbol{n}_{\Omega}=0$ on $\partial \Omega$. For all $c \in \mathcal{C}$, the equivalent of the component flux reconstruction $\Theta_{c, h}^{n}$ from Section 3.4 is then given by

$$
\boldsymbol{\Theta}_{c, h}^{n, k, i}:=\boldsymbol{\Theta}_{\mathrm{disc}, c, h}^{n, k, i}+\boldsymbol{\Theta}_{\mathrm{lin}, c, h}^{n, k, i}+\mathbf{\Theta}_{\mathrm{alg}, c, h}^{n, k, i} .
$$

Remark 4.3 (Algebraic error). In practice it is rather difficult to satisfy (4.14c exactly, though it is possible following, e.g., [46, Section 7.3]. Following [43, 41] and [36, Section 4] we prefer to compute our algebraic error flux reconstructions $\Theta_{\text {alg,c,h }}^{n, k, i}$ by: (i) performing $j$ additional iterations of the algebraic solver from the stage (4.3), with $j$ a user-defined fixed number; (ii) computing $\boldsymbol{\Theta}_{\mathrm{disc}, c, h}^{n, k, i+j}$ and $\boldsymbol{\Theta}_{\mathrm{lin}, c, h}^{n, k, j}$ as in (4.14a and 4.14b), respectively, with $i$ replaced by $i+j$; (iii) defining the algebraic error flux reconstruction as $\boldsymbol{\Theta}_{\mathrm{alg}, c, h}^{n, k, h}:=\boldsymbol{\Theta}_{\mathrm{disc}, c, h}^{n, k, i+j}+\boldsymbol{\Theta}_{\mathrm{lin}, c, h}^{n, k, i+j}-\left(\boldsymbol{\Theta}_{\mathrm{disc}, c, h}^{n, k, i}+\boldsymbol{\Theta}_{\mathrm{lin}, c, h}^{n, k, i}\right)$. Then, 4.14c only holds approximately, the better the bigger $j$ is. A rigorous analysis including an adaptive choice of the parameter $j$ has been carried out in [36]. In the numerical experiments below, a surprisingly good performance of this procedure is reported for the very low value $j=2$. This may be attributed to the use of an efficient preconditioning, possible without any change in the above considerations.

\subsection{Distinguishing the space, time, linearization, and algebraic errors}

In this section, we first give a time-localized version of Theorem 3.3. Subsequently, we derive an a posteriori error estimate distinguishing the space, time, linearization, and algebraic error components.

\subsubsection{A time-localized a posteriori error estimate}

Let $1 \leq n \leq N$, a Newton linearization iteration $k \geq 1$, and an algebraic solver iteration $i \geq 1$ be fixed. It follows from (4.8), the definition 4.14d of the flux reconstruction $\Theta_{c, h}^{n, k, i}$, and Green's theorem that there holds, for all $c \in \mathcal{C}$,

$$
\left(q_{c, h}^{n}-\frac{l_{c, M}\left(\mathcal{X}_{\mathcal{M}}^{n, k-1}\right)+\mathcal{L}_{c, M}^{n, k, i}-l_{c, M}^{n-1}}{\tau^{n}}-\nabla \cdot \Theta_{c, h}^{n, k, i}, 1\right)_{M}=0 \quad \forall M \in \mathcal{M}^{n} .
$$

Unfortunately, owing to the nonlinear accumulation term, compare the definition 4.12c) of $l_{c, h \tau}^{n, k, i}$ with 4.8), (4.15) is not a full equivalent of (3.10a). However, we can still elaborate Theorem 3.3 as follows. For all $c \in \mathcal{C}$, define the following refined version of the estimators of $(3.12)$, with the additional nonlinear accumulation estimator $\eta_{\mathrm{NA}, M, c}^{n, k, i}$ :

$$
\begin{aligned}
\eta_{\mathrm{R}, M, c}^{n, k, i} & :=\min \left\{C_{\mathrm{P}, M}, \varepsilon^{-\frac{1}{2}}\right\} h_{M}\left\|q_{c, h}^{n}-\left(\tau^{n}\right)^{-1}\left(l_{c, M}\left(\mathcal{X}_{\mathcal{M}}^{n, k-1}\right)+\mathcal{L}_{c, M}^{n, k, i}-l_{c, M}^{n-1}\right)-\nabla \cdot \Theta_{c, h}^{n, k, i}\right\|_{M}, \\
\eta_{\mathrm{F}, M, c}^{n, k, i}(t) & :=\left\|\boldsymbol{\Theta}_{c, h}^{n, k, i}-\boldsymbol{\Phi}_{c, h \tau}^{n, k, i}(t)\right\|_{M} \quad t \in I_{n}, \\
\eta_{\mathrm{NC}, M, p, c}^{n, k, i}(t) & :=\left\|\boldsymbol{\Psi}_{p, c}\left(P_{p, h \tau}^{n, k, i}\right)(t)-\boldsymbol{\Psi}_{p, c}\left(\mathfrak{P}_{p, h \tau}^{n, k, i}\right)(t)\right\|_{M} \quad t \in I_{n}, p \in \mathcal{P}_{c}, \\
\eta_{\mathrm{NA}, M, c}^{n, i}, & :=\varepsilon^{-\frac{1}{2}} h_{M}\left(\tau^{n}\right)^{-1}\left\|l_{c, M}\left(\mathcal{X}_{\mathcal{M}}^{n, k, i}\right)-l_{c, M}\left(\mathcal{X}_{\mathcal{M}}^{n, k-1}\right)-\mathcal{L}_{c, M}^{n, k, i}\right\|_{M},
\end{aligned}
$$

where the functions $\boldsymbol{\Psi}_{p, c}, p \in \mathcal{P}, c \in \mathcal{C}_{p}$, are defined by (3.9), while

$$
\boldsymbol{\Phi}_{c, h \tau}^{n, k, i}:=\sum_{p \in \mathcal{P}_{c}} \boldsymbol{\Phi}_{p, c, h \tau}^{n, k, i}, \quad \boldsymbol{\Phi}_{p, c, h \tau}^{n, k, i}:=\nu_{p}\left(P_{p, h \tau}^{n, k, i}, \boldsymbol{S}_{h \tau}^{n, k, i}, \boldsymbol{C}_{p, h \tau}^{n, k, i}\right) C_{p, c, h \tau}^{n, k, i} \boldsymbol{v}_{p}\left(P_{p, h \tau}^{n, k, i}, \boldsymbol{C}_{p, h \tau}^{n, k, i}\right) .
$$


In the spirit of Section 3.3 , we define the time-localized error measure,

$$
\mathcal{N}^{n}:=\left\{\sum_{c \in \mathcal{C}}\left(\mathcal{N}_{c}^{n}\right)^{2}\right\}^{\frac{1}{2}}+\left\{\sum_{p \in \mathcal{P}}\left(\mathcal{N}_{p}^{n}\right)^{2}\right\}^{\frac{1}{2}}
$$

where $\mathcal{N}_{c}^{n}, c \in \mathcal{C}$, and $\mathcal{N}_{p}^{n}, p \in \mathcal{P}$, are defined as $(3.6)$ and $(3.8)$, respectively, with the current approximations indexed $n, k, i$ and the time integration performed on the time intervals $I_{n}$ instead of $\left(0, t_{\mathrm{F}}\right)$. Note that

$$
\mathcal{N}_{c}=\sum_{n=1}^{N}\left(\mathcal{N}_{c}^{n}\right)^{2}, \quad \mathcal{N}_{p}=\sum_{n=1}^{N}\left(\mathcal{N}_{p}^{n}\right)^{2}
$$

We then have:

Corollary 4.4 (Time-localized a posteriori error estimate). Consider a time step $1 \leq n \leq N$, a Newton linearization iteration $k \geq 1$, and an algebraic solver iteration $i \geq 1$. Under Assumption 3.1, for the approximate solution of Section 4.2. the phase pressure reconstructions of Section 4.3, the component flux reconstructions of Section 4.4. and with the estimators given by (4.16), there holds

$$
\begin{aligned}
\mathcal{N}_{c}^{n} \leq\left\{\int_{I_{n}} \sum_{M \in \mathcal{M}^{n}}\left(\eta_{\mathrm{R}, M, c}^{n, k, i}+\eta_{\mathrm{F}, M, c}^{n, k, i}(t)+\eta_{\mathrm{NA}, M, c}^{n, k, i}\right)^{2} \mathrm{~d} t\right\}^{\frac{1}{2}} \quad c \in \mathcal{C}, \\
\mathcal{N}_{p}^{n} \leq\left\{\sum_{c \in \mathcal{C}_{p}} \int_{I_{n}} \sum_{M \in \mathcal{M}^{n}}\left(\eta_{\mathrm{NC}, M, p, c}^{n, k, i}(t)\right)^{2} \mathrm{~d} t\right\}^{\frac{1}{2}} \quad p \in \mathcal{P} .
\end{aligned}
$$

Proof. The proof is a slight modification of that of Theorem 3.3 . We only need to estimate

$$
\begin{aligned}
& \sum_{n=1}^{N} \int_{I_{n}} \sum_{M \in \mathcal{M}^{n}}\left(\partial_{t}^{n} l_{c, h \tau}^{n, k, i}-\left(\tau^{n}\right)^{-1}\left(l_{c, M}\left(\mathcal{X}_{\mathcal{M}}^{n, k-1}\right)+\mathcal{L}_{c, M}^{n, k, i}-l_{c, M}^{n-1}\right), \varphi\right)_{M}(t) \mathrm{d} t \\
= & \sum_{n=1}^{N} \int_{I_{n}} \sum_{M \in \mathcal{M}^{n}}\left(\left(\tau^{n}\right)^{-1}\left(l_{c, M}\left(\mathcal{X}_{\mathcal{M}}^{n, k, i}\right)-l_{c, M}\left(\mathcal{X}_{\mathcal{M}}^{n, k-1}\right)-\mathcal{L}_{c, M}^{n, k, i}\right), \varphi\right)_{M}(t) \mathrm{d} t \\
\leq & \sum_{n=1}^{N} \int_{I_{n}} \sum_{M \in \mathcal{M}^{n}} \eta_{\mathrm{NA}, M, c^{n}}^{n, k, i} \varepsilon^{\frac{1}{2}} h_{M}^{-1}\|\varphi\|_{M}(t) \mathrm{d} t \leq \sum_{n=1}^{N} \int_{I_{n}} \sum_{M \in \mathcal{M}^{n}} \eta_{\mathrm{NA}, M, c}^{n, k, i}\|\varphi\|_{X, M}(t) \mathrm{d} t,
\end{aligned}
$$

to combine this bound with 3.14 and the definition 3.2 of the norm on the space $X$, and restrict the result to the given time interval.

\subsubsection{Distinguishing the different error components}

For all $1 \leq n \leq N, k \geq 1, i \geq 1, M \in \mathcal{M}^{n}$, and $c \in \mathcal{C}$, we define the spatial estimators evaluating the error related to the spatial mesh resolution,

$$
\eta_{\mathrm{sp}, M, c}^{n, k, i}(t):=\eta_{\mathrm{R}, M, c}^{n, k, i}+\left\|\Theta_{\mathrm{disc}, c, h}^{n, k, i}-\boldsymbol{\Phi}_{c, h \tau}^{n, k, i}\left(t^{n}\right)\right\|_{M}+\left\{\sum_{p \in \mathcal{P}_{c}}\left(\eta_{\mathrm{NC}, M, p, c}^{n, k, i}(t)\right)^{2}\right\}^{\frac{1}{2}} \quad t \in I_{n}
$$

the temporal estimators evaluating the error related to the size of the time step,

$$
\eta_{\mathrm{tm}, M, c}^{n, k, i}(t):=\left\|\boldsymbol{\Phi}_{c, h \tau}^{n, k, i}\left(t^{n}\right)-\boldsymbol{\Phi}_{c, h \tau}^{n, k, i}(t)\right\|_{M} \quad t \in I_{n}
$$

the linearization estimators measuring the error in the linearization of the nonlinear system (4.1),

$$
\eta_{\operatorname{lin}, M, c}^{n, k, i}:=\left\|\Theta_{\operatorname{lin}, c, h}^{n, k, i}\right\|_{M}+\eta_{\mathrm{NA}, M, c}^{n, k, i}
$$

and the algebraic estimators that quantify the error in the algebraic iterative resolution of the linear system 4.2,

$$
\eta_{\mathrm{alg}, M, c}^{n, k, i}:=\left\|\Theta_{\mathrm{alg}, c, h}^{n, k, i}\right\|_{M} .
$$


For all $c \in \mathcal{C}$, global versions of these estimators are given by

$$
\begin{aligned}
\eta_{\mathrm{sp}, c}^{n, k, i} & :=\left\{4 \int_{I_{n}} \sum_{M \in \mathcal{M}^{n}}\left(\eta_{\mathrm{sp}, M, c}^{n, k, i}(t)\right)^{2} \mathrm{~d} t\right\}^{\frac{1}{2}}, \\
\eta_{\mathrm{tm}, c}^{n, k, i} & :=\left\{2 \int_{I_{n}} \sum_{M \in \mathcal{M}^{n}}\left(\eta_{\mathrm{tm}, M, c}^{n, k, i}(t)\right)^{2} \mathrm{~d} t\right\}^{\frac{1}{2}}, \\
\eta_{\mathrm{lin}, c}^{n, k, i} & :=\left\{2 \tau^{n} \sum_{M \in \mathcal{M}^{n}}\left(\eta_{\mathrm{lin}, M, c}^{n, k, i}\right)^{2}\right\}^{\frac{1}{2}}, \\
\eta_{\mathrm{alg}, c}^{n, k, i} & :=\left\{2 \tau^{n} \sum_{M \in \mathcal{M}^{n}}\left(\eta_{\mathrm{alg}, M, c}^{n, k, i}\right)^{2}\right\}^{\frac{1}{2}} .
\end{aligned}
$$

Using the triangle inequality and Corollary 4.4 , we can estimate the time-localized norm $\mathcal{N}^{n}$ of 4.17 as follows:

Corollary 4.5 (Distinguishing the space, time, linearization, and algebraic errors). Under the assumptions of Corollary 4.4, there holds, with the estimators given by 4.20,

$$
\mathcal{N}^{n} \leq\left\{\sum_{c \in \mathcal{C}}\left(\eta_{\mathrm{sp}, c}^{n, k, i}+\eta_{\mathrm{tm}, c}^{n, k, i}+\eta_{\mathrm{lin}, c}^{n, k, i}+\eta_{\mathrm{alg}, c}^{n, k, i}\right)^{2}\right\}^{\frac{1}{2}}
$$

Remark 4.6 (Anisotropic meshes and full permeability tensors). For more general polygonal or polyhedral meshes and for full permeability tensors, the accuracy of RTN flux reconstructions may deteriorate. As one alternative, the choice of the norm 3.2 can be adapted to account for anisotropies and inhomogeneities.

\subsection{A fully adaptive algorithm}

In this section we propose an adaptive algorithm based on the estimators 4.20 . Let $\gamma_{\operatorname{lin}}, \gamma_{\text {alg }} \in(0,1)$ be user-given parameters; these express respectively the fraction allowed for the linearization and algebraic error components. Similarly, let the parameters for balancing the spatial and temporal errors $\Gamma_{\mathrm{tm}}>$ $\gamma_{\mathrm{tm}}>0$ be fixed. Finally, let $\operatorname{crit}_{c}^{n}$ stand for the maximal error allowed in the component $c$ on the time interval $I_{n}$. Our algorithm is as follows:

\section{Initialization}

(a) Choose an initial mesh $\mathcal{M}^{0}$, an initial time step $\tau^{0}$, and set $t^{0}:=0$ and $n:=0$.

(b) Set up the initial approximation $\mathcal{X}_{\mathcal{M}}^{0}$.

\section{Loop in time}

(a) Set $n:=n+1, \mathcal{M}^{n}:=\mathcal{M}^{n-1}, \tau^{n}:=\tau^{n-1}$, and $k:=0$.

(b) Define $\mathcal{X}_{\mathcal{M}}^{n, 0}:=\mathcal{X}_{\mathcal{M}}^{n-1}$.

(c) Spatial and temporal errors balancing loop

\section{i. Newton linearization loop}

\section{A. Newton initialization}

- Set $k:=k+1$ and $i:=0$.

- Define $\mathcal{X}_{\mathcal{M}}^{n, k, 0}:=\mathcal{X}_{\mathcal{M}}^{n, k-1}$.

B. Set up the linear system.

- Compute the linearized component fluxes $F_{c, M, \sigma}^{n, k, i}, c \in \mathcal{C}$, following 4.6.). 
- Compute the perturbation of the accumulation term $\mathcal{L}_{c, M}^{n, k, i}, c \in \mathcal{C}$, following (4.5).

- Assemble the linear system following (4.8).

C. Algebraic solver loop

- Set $i:=i+1$.

- Perform a step of the iterative algebraic solver for the solution of (4.8).

- A posteriori estimators

- Build the postprocessed phase pressures following 4.11.

- Construct the approximations of the saturations, molar fractions, and amounts of components following 4.12 .

- Prescribe the continuous phase pressure reconstructions following 4.13.

- Construct the component flux reconstructions $\boldsymbol{\Theta}_{\mathrm{disc}, c, h}^{n, k, i}, \boldsymbol{\Theta}_{\operatorname{lin}, c, h}^{n, k, i}, \mathbf{\Theta}_{\mathrm{alg}, c, h}^{n, k, i}$, and $\boldsymbol{\Theta}_{c, h}^{n, k, i}$ following 4.14).

- Evaluate the different estimators defined by 4.20 ).

- Terminate the algebraic solver loop if

$$
\eta_{\text {alg }}^{c} n, k, i \leq \gamma_{\text {alg }}\left(\eta_{\mathrm{sp}}^{c} n, k, i+\eta_{\mathrm{tm}}^{c} n, k, i+\eta_{\operatorname{lin}}^{c} n, k, i\right), \quad \forall c \in \mathcal{C} .
$$

D. Update

- Update the unknowns; set $\mathcal{X}_{\mathcal{M}}^{n, k}:=\mathcal{X}_{\mathcal{M}}^{n, k, i}$.

E. Terminate the Newton linearization loop if

$$
\eta_{\text {lin }}^{c} n, k, i \leq \gamma_{\operatorname{lin}}\left(\eta_{\mathrm{sp}}^{c} n, k, i+\eta_{\mathrm{tm}}^{c} n, k, i\right), \quad \forall c \in \mathcal{C} .
$$

ii. Adapt the time step if necessary.

iii. If spatial mesh adaptation is considered, refine or coarsen the mesh $\mathcal{M}^{n}$ in function of the distribution of the local spatial error estimators $\eta_{\mathrm{sp}, M, c}^{n, k, i}(t)$ of $4.19 \mathrm{a}$.

iv. Terminate the spatial and temporal errors balancing loop if

$$
\gamma_{\mathrm{tm}} \eta_{\mathrm{sp}}^{c} n, k, i \leq \eta_{\mathrm{tm}}^{c} n, k, i \leq \Gamma_{\mathrm{tm}} \eta_{\mathrm{sp}}^{c} n, k, i \quad \text { and } \quad \eta_{\mathrm{sp}}^{c} n, k, i+\eta_{\mathrm{tm}}^{c} n, k, i \leq \mathrm{crit}_{c}^{n} \quad \forall c \in \mathcal{C} .
$$

\section{(d) Data update}

$$
\text { i. Set } \mathcal{X}_{\mathcal{M}}^{n}:=\mathcal{X}_{\mathcal{M}}^{n, k, i} \text { and } t^{n}:=t^{n-1}+\tau^{n} \text {. }
$$

(e) End: Loop in time if $\left(t^{n}>t^{F}\right)$.

Note that in 4.22 we propose to stop the iterative algebraic solver when the algebraic error components do not affect significantly the overall error. A variation in the spirit of Remark 4.3 can be considered where a user-defined number $j$ of linear iterations are performed before recomputing the estimators, and the algebraic estimator is replaced by its approximate version. Similarly, the criterion 4.23 expresses the fact that there is no need to continue with the linearization iterations if the overall error is dominated by the space and time errors. Finally, by 4.24 we give a way to select the time step $\tau^{n}$ in order to equilibrate the space and time error components; congruently, the spatial mesh should be refined/derefined. If local adaptive mesh refinement is considered, $\mathcal{M}^{n}$ should be such that, for all $M_{1}, M_{2} \in \mathcal{M}^{n}$ with $M_{1} \neq M_{2}$,

$$
\eta_{\mathrm{sp}, c, M_{1}}^{n, k, i} \approx \eta_{\mathrm{sp}, c, M_{2}}^{n, k, i}, \quad \forall c \in \mathcal{C} .
$$

Local (elementwise) versions of the criteria 4.22 and 4.23 can be formulated using the local estimators 4.19; ; see [46, 33, 36].

Remark 4.7 (Mass balance and stopping criteria). Our a posteriori analysis is based on the reconstruction of $N_{\mathcal{C}}$ conservative component fluxes, see (3.10a) in Section 3.4 and 4.15) in Section 4.5. We achieve (3.10a) in a straightforward way if a) the accumulation term $l_{c}$ of $(2.5)$ is linear and $b$ ) the arising algebraic systems are solved "exactly" (up to machine precision) or if 4.14c) is satisfied. Then, on each step of the algorithm of Section 4.6, we have full mass conservation regardless of the value of the parameters $\gamma_{\mathrm{lin}}$ and $\gamma_{\mathrm{alg}}$. Unfortunately, for nonlinear accumulation terms and for the construction of the algebraic error fluxes of Remark 4.3. the mass balance conservation may be violated. A rigorous analysis of the error related to the mass balance violation by the approach of Remark 4.3 is done in [36]. In the numerical results below we study the mass balance violation in our adaptive resolution and compare it to the classical one. 


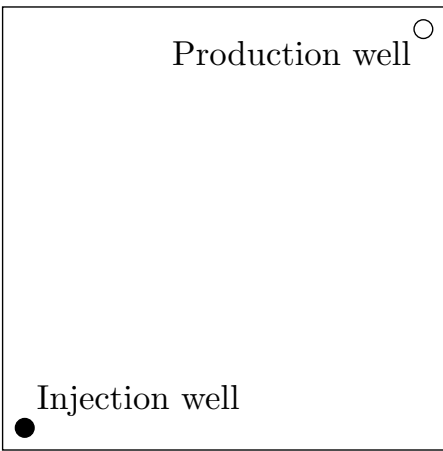

(a) Two-spot pattern

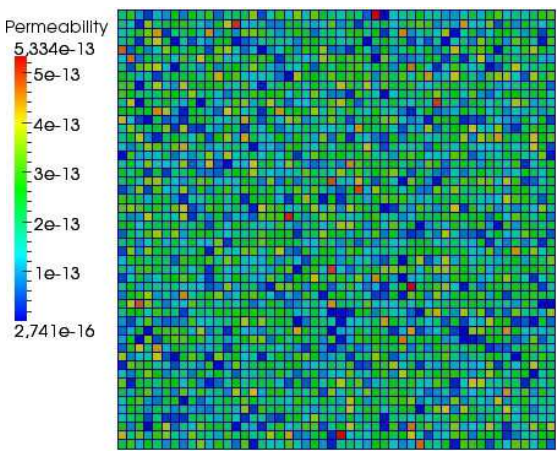

(b) Absolute permeability for the heterogeneous test case of Section 5.3

Figure 1: Configuration for the numerical test cases

\section{$5 \quad$ Numerical results}

In this section we illustrate our theoretical results on two test cases representative of enhanced oil recovery techniques. More specifically, we focus on the case when oil recovery is improved by injecting components that are not originally present in the reservoir to increase the mobility, cf., e.g., [53, 62. Both homogeneous and heterogeneous (but isotropic) porous media are considered.

\section{$5.1 \quad$ Setting}

We consider the injection of gas composed of carbon dioxide $\mathrm{CO}_{2}$ (component $\mathrm{g}_{1}$ ) and nitrogen $\mathrm{N}_{2}$ (component $\mathrm{g}_{2}$ ) into a reservoir initially saturated with heptanol $\mathrm{C}_{7} \mathrm{H}_{16}$ (component o). The three components, collected in the set $\mathcal{C}:=\left\{\mathrm{o}, \mathrm{g}_{1}, \mathrm{~g}_{2}\right\}$, can be present in a liquid or gaseous phase corresponding to $\mathcal{P}:=\{l, g\}$. This is therefore a special case of the more general problem considered in Example 2 The spatial domain is $\Omega=(0,1000) \mathrm{m} \times(0,1000) \mathrm{m}$ and the process is simulated for $t_{\mathrm{F}}=7$ years. We consider a two-spot pattern, see Figure 1a, where we have one injection well with pressure fixed to $P_{\text {inj }}=1.1 \times 10^{10} \mathrm{~Pa}$ and one production well with pressure fixed to $P_{\text {pro }}=9 \times 10^{6} \mathrm{~Pa}$. Wells are modeled as nonlinear source terms as detailed in the following. Denoting by $M_{\text {inj }}$ and $M_{\text {pro }}$ the cells containing the injection and production wells, respectively, the rates of injection and production of the component $c \in \mathcal{C}$, denoted by $q_{c, M_{\mathrm{inj}}}$ and $q_{c, M_{\mathrm{pro}}}$, are given by the following expressions:

$$
\left|M_{\mathrm{inj}}\right| q_{c, M_{\mathrm{inj}}}=-\nu_{\mathrm{g}, M_{\mathrm{inj}}}\left(P_{\mathrm{inj}}, S_{\mathrm{g}, \mathrm{inj}}, \boldsymbol{C}_{\mathrm{g}, \mathrm{inj}}\right) C_{\mathrm{g}, c, \mathrm{inj}} \mathrm{IP}_{M_{\mathrm{inj}}}\left(P_{\mathrm{g}, M_{\mathrm{inj}}}-P_{\mathrm{inj}}\right)
$$

and

$$
\left|M_{\text {pro }}\right| q_{c, M_{\text {pro }}}=-\sum_{p \in \mathcal{C}_{p}}\left\{\nu_{p, M_{\text {pro }}}\left(P_{p, M_{\text {pro }}}, S_{p, M_{\text {pro }}}, C_{p, M_{\text {pro }}}\right) C_{p, c, M_{\text {pro }}} \operatorname{IP}_{M_{\text {pro }}}\left(P_{p, M_{\text {pro }}}-P_{\text {pro }}\right)\right\}
$$

where $S_{\mathrm{g}, \text { inj }}=1$ is the injected gas saturation, $\boldsymbol{C}_{\mathrm{g}, \text { inj }}$ is the vector of injected components molar fractions in gas

$$
C_{\mathrm{g}, \mathrm{inj}}=\left\{C_{\mathrm{g}, \mathrm{o}, \mathrm{inj}}=0, C_{\mathrm{g}, \mathrm{g}_{1}, \mathrm{inj}}=0.8, C_{\mathrm{g}, \mathrm{g}_{2}, \mathrm{inj}}=0.2\right\},
$$

and $\mathrm{IP}_{M}$ is the well's production index given, for isotropic medium, by Peaceman's formula:

$$
\mathrm{IP}_{M}:=\frac{2 \pi \lambda_{M} \Delta Z_{M}}{\log \left(0.14 \frac{r_{\mathrm{d}}}{r_{\mathrm{w}}}\right)}, \quad r_{\mathrm{d}}:=\sqrt{\Delta x_{M}^{2}+\Delta y_{M}^{2}},
$$

with $r_{\mathrm{w}}$ the well radius set to $0.5 \mathrm{~m}, r_{\mathrm{d}}$ the equivalent block well radius, $\Delta Z_{M}$ the perforated mesh height, and $\Delta x_{M}, \Delta y_{M}$ the linear dimensions of the perforated cell $M$ along the $x$ and $y$ axis, respectively. The required physical properties are chosen as follows: (i) porosity $\phi=0.1$. (ii) phase molar density $\zeta_{p}=\sum_{c \in \mathcal{C}} \zeta_{c}(P) C_{c, p}, p \in\{1, \mathrm{~g}\}$, where $\zeta_{c}, c \in \mathcal{C}$, takes the form

$$
\zeta_{c}(P)=\alpha_{c} \frac{P-P_{\text {inj }}}{P_{\text {pro }}-P_{\text {inj }}}+\beta_{c} \frac{P-P_{\text {pro }}}{P_{\text {inj }}-P_{\text {pro }}},
$$


with $\left(\alpha_{\mathrm{o}}, \beta_{\mathrm{o}}\right)=(6640.88,6669.32),\left(\alpha_{\mathrm{g}_{1}}, \beta_{\mathrm{g}_{2}}\right)=(4703.4,5567.0)$, and $\left(\alpha_{\mathrm{g}_{2}}, \beta_{\mathrm{g}_{2}}\right)=(3062.5,3676.4)$. (iii) The liquid phase viscosity $\mu_{\mathrm{l}}=3.2 \times 10^{-4} \mathrm{Pas}$, the gas phase viscosity $\mu_{\mathrm{g}}=3.5 \times 10^{-5} \mathrm{Pas}$. (iv) The relative permeability

$$
k_{\mathrm{r}, \mathrm{p}}\left(S_{p}\right)= \begin{cases}1 & \text { if } S_{p} \geq 1, \\ \frac{S_{p}-S_{p}^{\text {res }}}{1-S_{p}^{\text {res }}} & \text { if } S_{p}^{\text {res }}<S_{p}<1, \\ 0 & \text { if } S_{p} \leq S_{p}^{\text {res }},\end{cases}
$$

where the residual saturations are respectively given by $S_{1}^{\text {res }}=0.2$ and $S_{\mathrm{g}}^{\text {res }}=0.1$. Concerning the thermodynamic equilibrium between the oil and gas phases

$$
C_{\mathrm{g}, c}=K_{c}^{\mathrm{o}, \mathrm{g}}\left(P, \boldsymbol{C}_{\mathrm{o}}, \boldsymbol{C}_{\mathrm{g}}\right) C_{\mathrm{o}, c}, \quad \forall c \in \mathcal{C},
$$

we consider here a simple formula, depending just on the pressure, for the equilibrium constant $K_{c}^{\text {o,g }}=$ $K_{c}^{\mathrm{o}, \mathrm{g}}(P)$ between the oil and gas phases for the component $c \in \mathcal{C}$ given by

$$
K_{c}^{\mathrm{o}, \mathrm{g}}(P)=\gamma_{c} \frac{P-P_{\text {inj }}}{P_{\text {pro }}-P_{\text {inj }}}+\delta_{c} \frac{P-P_{\text {pro }}}{P_{\text {inj }}-P_{\text {pro }}},
$$

with $\left(\gamma_{\mathrm{o}}, \delta_{\mathrm{o}}\right)=\left(1.2 \times 10^{-2}, 1 \times 10^{-2}\right),\left(\gamma_{\mathrm{g}_{1}}, \delta_{\mathrm{g}_{1}}\right)=\left(1.3 \times 10^{1}, 1.64 \times 10^{1}\right)$, and $\left(\gamma_{\mathrm{g}_{2}}, \delta_{\mathrm{g}_{2}}\right)=(64,76)$. Note that, as we consider a horizontal $2 \mathrm{D}$ case, gravitational effects are not taken into account in the numerical tests, and the phase mass densities $\rho_{p}, p \in \mathcal{P}$, need not be specified. We shall test two cases, with a homogeneous porous medium and a heterogeneous one. The capillary pressure curves and the absolute permeability $\boldsymbol{\Lambda}$ are problem-specific and will be described below.

We consider a uniform spatial mesh (mesh adaptation will be considered in a future work) and choose the initial time step as $\tau^{0}=5.184 \times 10^{5} \mathrm{~s}$, which equals to 6 days. We consider the finite volume discretization of Section 2.2.2 with the Newton linearization detailed in Section 4.1. we obtain (4.8) with the GMRes iterative solver and ILU preconditioner with zero level fill-in. Our implementation uses PETSc [12, 11, 13] with the function KSPSetConvergenceTest allowing to enter a user-defined convergence criterion.

In order to compare the adaptive resolution with a classical one, we introduce the relative residuals related to the linearization and algebraic resolutions. Consider, at every time step $n$, a resolution of a system of nonlinear algebraic equations $F^{n}(X)=0$ by the Newton method. Starting from $X^{n, 0}$, the relative linearization residual at step $k$ is defined as

$$
\operatorname{err}_{\operatorname{lin}}^{n, k}:=\frac{\left\|F^{n}\left(X^{n, k}\right)\right\|}{\left\|F^{n}\left(X^{n, 0}\right)\right\|}
$$

Similarly, consider the linear system resulting from the Newton method and written in the following form: $A^{n, k-1} X^{n, k}=b^{n, k-1}$. An iterative algebraic solver for this linear system looks at each step $i$ for a vector $X^{n, k, i}$ whose relative algebraic residual is expressed by

$$
\operatorname{err}_{\text {alg }}^{n, k, i}:=\frac{\left\|A^{n, k-1} X^{n, k, i}-b^{n, k-1}\right\|}{\left\|b^{n, k-1}\right\|} .
$$

The comparison in numerical tests below will be done between the adaptive resolution where the stopping criteria for the GMRes and Newton iterations are given by, respectively, 4.22 and 4.23 with $\gamma_{\text {alg }}=$ $\gamma_{\text {lin }}=10^{-3}$, and a classical algorithm where iterations are stopped using a fixed threshold, i.e.,

$$
\operatorname{err}_{\text {alg }}^{n, k, i} \leq 10^{-8},
$$

for the GMRes iterations and

$$
\operatorname{err}_{\text {lin }}^{n, k} \leq 10^{-8}
$$

for the Newton linearization. Please note the above relative residuals $\operatorname{err}_{\text {lin }}^{n, k}$ and err $_{\text {alg }}^{n, k, i}$ are not directly comparable to our linearization and algebraic estimators $\eta_{\operatorname{lin}, c}^{n, k}$ and $\eta_{\mathrm{alg}, c}^{n, k, i}$ given respectively by (4.20c) and (4.20d). Thus in particular the classical stopping criteria (5.1) and (5.2) do not mean that our estimators should be below the $10^{-8}$ threshold. The algebraic error flux reconstruction $\Theta_{\text {alg, }, c, h}^{n, k}$ is obtained in the spirit of Remark 4.3 with $j=2$. We thus perform two additional GMRes iterations before checking the criterion 4.22 (these additional steps are not wasted as we continue the iteration from the last obtained solution in the next GMRes step). 


\subsection{Compressible flow in a homogeneous porous medium}

We first consider a simplified test case with a homogeneous permeability $\boldsymbol{\Lambda}=\left(9.869233 \times 10^{-14} \mathrm{~m}^{2}\right) \mathbf{I}$, where $\mathbf{I}$ is the identity tensor, and no capillary effects, setting $P_{\mathbf{c}_{p}}(\boldsymbol{S}) \equiv 0$ for all $p \in \mathcal{P}$. For a fixed time step and the first Newton iteration, we first show in the left part of Figure 2 the evolution of the different estimators as a function of the GMRes iterations at the classical resolution stopped following (5.1). We remark that the algebraic error steadily decreases, while the other components stagnate starting from the first iteration. For the same time step, in the right part of Figure 2 we depict the evolution of the spatial, temporal, and linearization error estimators as a function of the number of Newton iterations. The spatial and temporal errors stagnate starting from the third step while the linearization error $\eta_{\text {lin, } M}^{n, k, i}$ decreases until $10^{-6}$, which is equivalent to the value $10^{-8}$ for the relative linearization residual at which we satisfy the classical stopping criterion (5.2). These results justify our stopping criteria which economize many useless iterations.

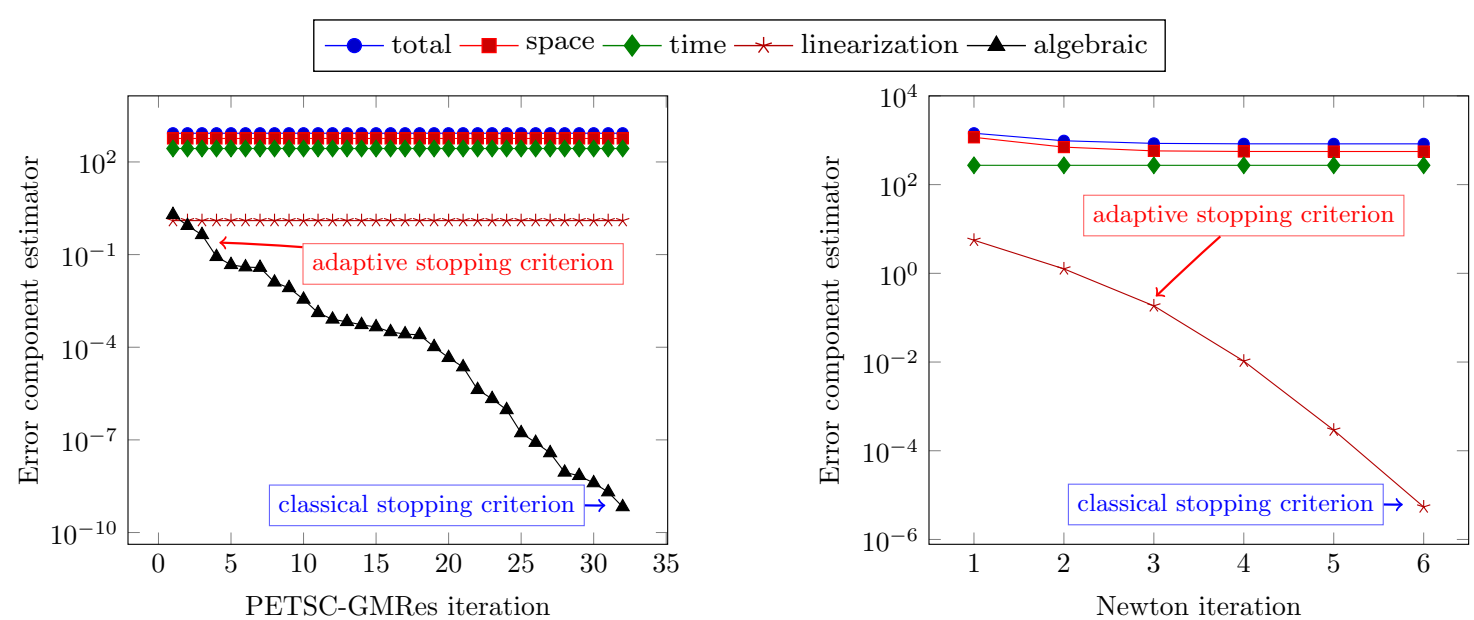

Figure 2: Evolution of the spatial, temporal, linearization, and algebraic error estimators 4.20 for a fixed mesh at time $1.04 \cdot 10^{6} \mathrm{~s}$, as a function of GMRes iterations on the first Newton iteration (left) and of Newton iterations (right)
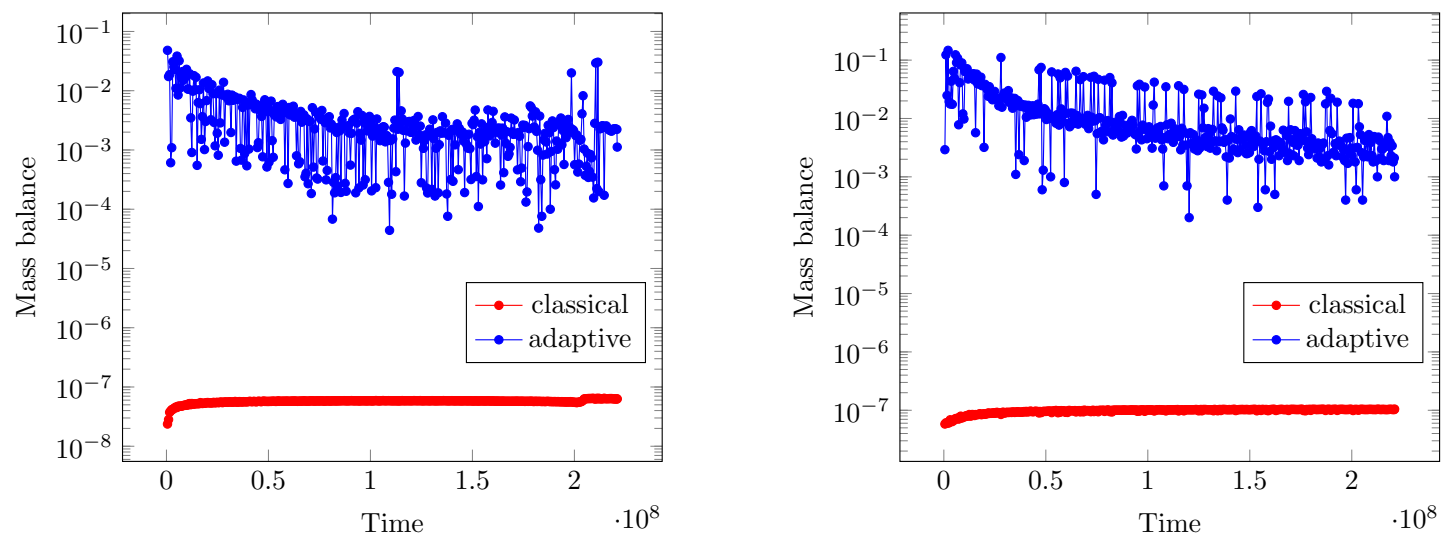

Figure 3: Evolution of mass balance violation for the test case of Section 5.2 (left) and for the test case of Section 5.3 (right)

Figure 3 illustrates the evolution of the mass balance violation due to the inexact algebraic resolution discussed in Remark 4.7. The violation of the mass balance at each time step is measured by the quantity 

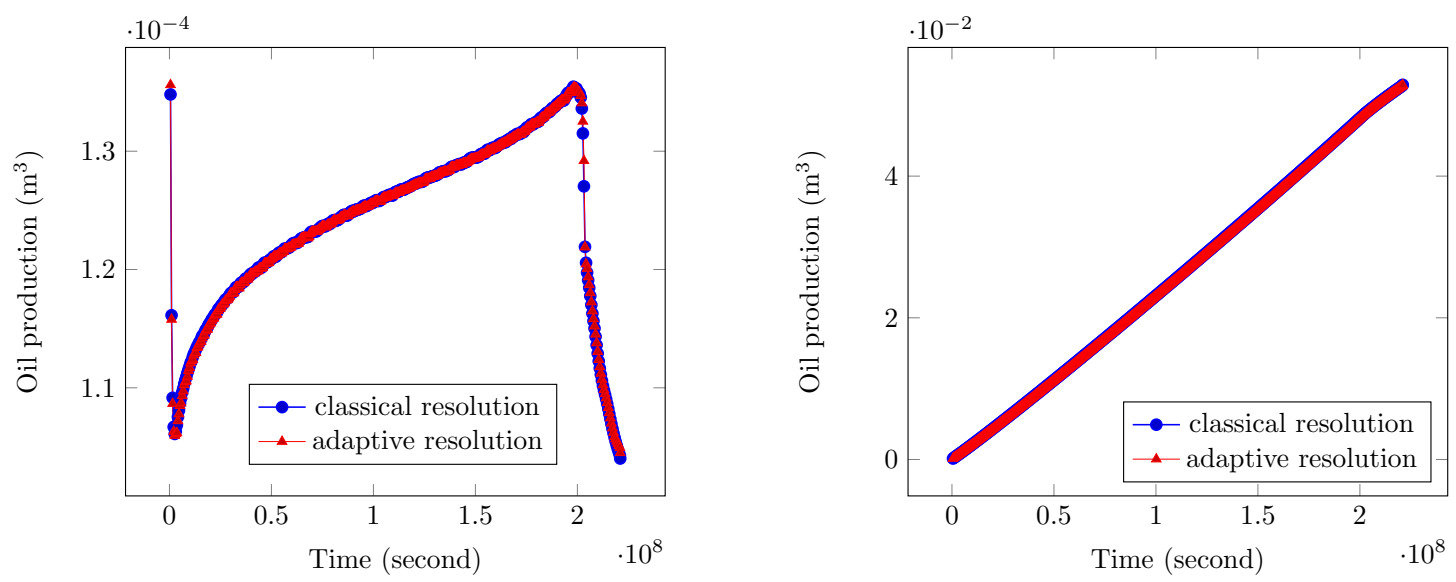

Figure 4: Instantaneous (left) and cumulated oil production (right) during the simulation, classical resolution vs. adaptive resolution for the test case of Section 5.2
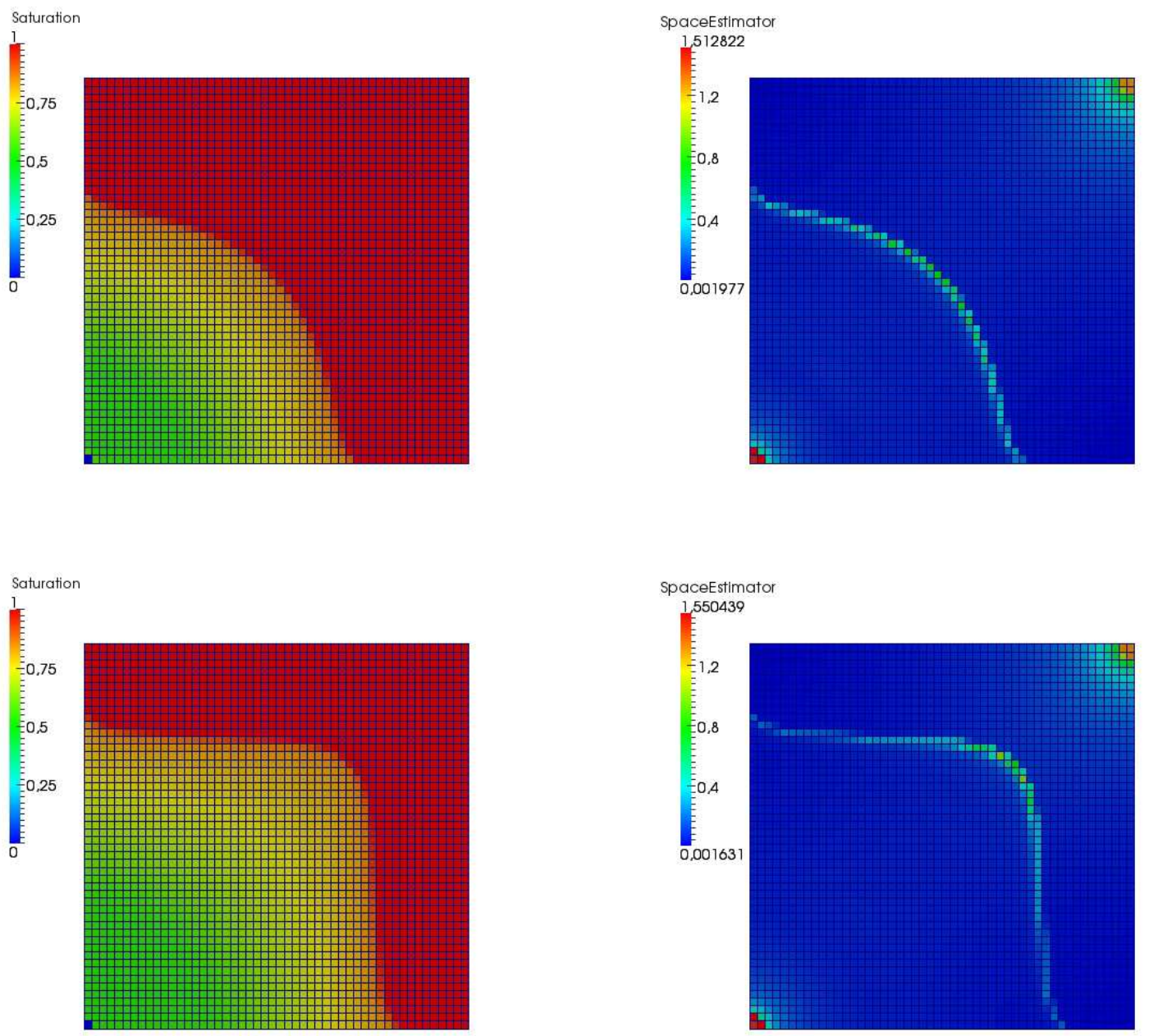

Figure 5: Liquid saturation (left) and distribution of the spatial estimators (right) for the adaptive resolution at times $7.8 \times 10^{7} \mathrm{~s}$ and $2.1 \times 10^{8} \mathrm{~s}$, the test case of Section 5.2 


$$
\eta_{\mathrm{MB}}^{n}:=\left\{\sum_{c \in \mathcal{C}}\left(\eta_{\mathrm{MB}, c}^{n}\right)^{2}\right\}^{\frac{1}{2}}, \quad \eta_{\mathrm{MB}, c}^{n}:=\left\{\int_{I_{n}} \sum_{M \in \mathcal{M}^{n}}\left\|q_{c, h}^{n}-\partial_{t}^{n} l_{c, h \tau}-\nabla \cdot \Theta_{c, h}^{n}\right\|_{M}^{2} \mathrm{~d} t\right\}^{\frac{1}{2}}, \quad c \in \mathcal{C} .
$$

We observe in the left part of Figure 3 that the adaptive resolution yields mass balance to an average precision of 2-3 digits, while the classical resolution provides on average 7 digits.Figure 4 shows on the left the instantaneous oil production at each time step, and on the right the cumulated oil production during the simulation for the classical and adaptive resolutions. For those crucial outputs, no sensible loss of the precision is observed due to the use of the adaptive algorithm. We next in Figure 5 show the evolution of the saturation in the liquid phase and the corresponding spatial estimators 4.19a for the oil component at two selected time values during the simulation. We see that this estimator detects the error caused by the two wells, as well as the error following the saturation front. This result pleads for a space mesh refinement/coarsening strategy using our estimators which will be considered in a future work.
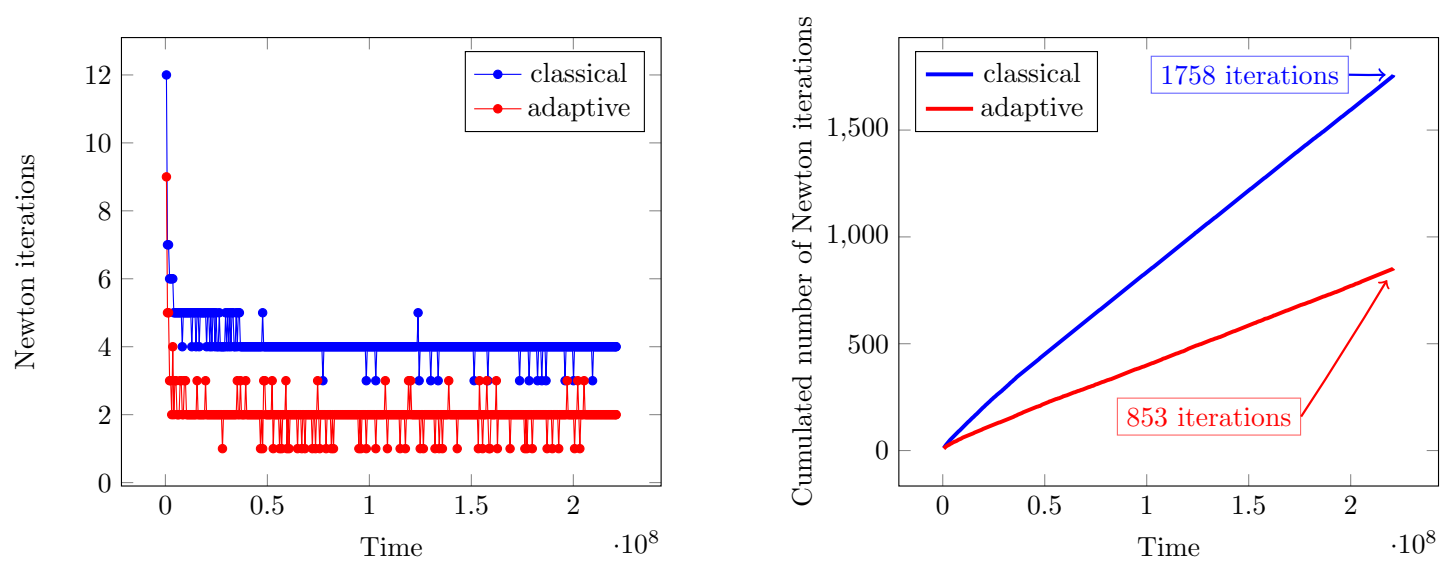

Figure 6: Newton iterations at each time step (left) and cumulated number of Newton iterations as a function of time (right) for the test case of Section 5.2. Average number of Newton iterations per time step: 4 iterations (classical), 2 iterations (adaptive)
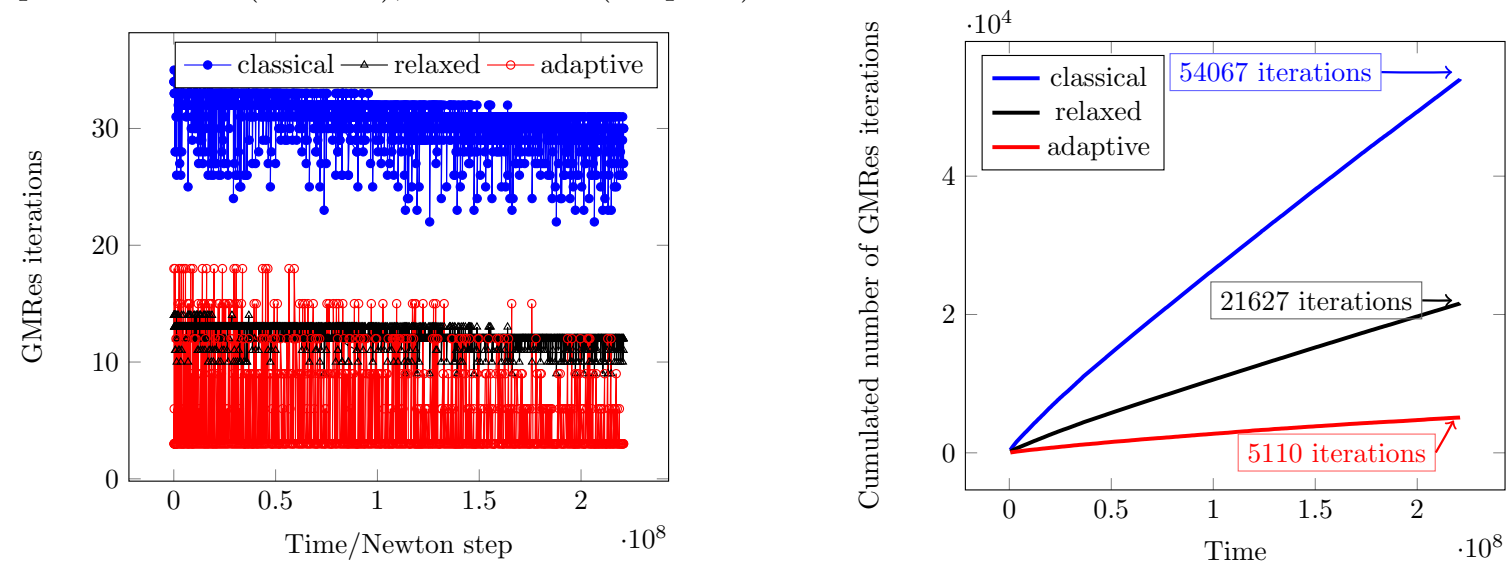

Figure 7: GMRes iterations for each time and Newton iteration step (left) and cumulated number of GMRes iterations as a function of time (right) for the test case of Section 5.2. Average number of GMRes iterations per time and Newton iteration step: 31 iterations (classical), 12 iterations (relaxed), 6 iterations (adaptive). Average number of GMRes iterations per time step: 126 iterations (classical), 51 iterations (relaxed), 12 iterations (adaptive)

We next focus on computational savings resulting from our adaptive stopping criteria. In the left part of Figure 6, we show the number of Newton iterations at each time step after the total simulation. 
The cumulated number of Newton iterations as a function of time is then presented in the right part of Figure 6. The overall gain in terms of linearization iterations obtained using our stopping criteria is quite significant.

To evaluate the influence of the algebraic stopping criterion, we compare the classical criterion (5.1), the adaptive criterion 4.22 , and a relaxed criterion

$$
\operatorname{err}_{\text {alg }}^{n, k, i} \leq 10^{-3} .
$$

Criterion (5.4) is representative of choices sometimes made in industrial simulators to speed-up the calculations. We represent in the left part of Figure 7 the number of GMRes iterations at each time and Newton step. In the right part of Figure 7, we then depict the cumulated number of GMRes iterations as a function of time steps. The gain in GMRes iterations reaches a factor of roughly 10 for the adaptive resolution compared with the classical one, and a factor of 4.2 compared with the relaxed one.

\subsection{Compressible flow in a heterogeneous porous medium}

We consider here the heterogeneous permeability field shown in Figure $1 \mathrm{~b}$ corresponding to a log-normal distribution. For the phase pressures, we choose $P_{1}$ as the reference pressure $P$, i.e. $P_{c_{1}} \equiv 0$, and follow the Brooks-Corey model [18] for the gas phase capillary pressure law, i.e.,

$$
P_{\mathrm{g}}=P+P_{c_{\mathrm{g}}}\left(S_{\mathrm{g}}\right), \quad P_{c_{\mathrm{g}}}\left(S_{\mathrm{g}}\right)=P_{e} \cdot\left(S_{e}\right)^{m}, \quad S_{e}=1-\frac{S_{\mathrm{g}}-S_{\mathrm{g}}^{\mathrm{res}}}{1-S_{\mathrm{l}}^{\text {res }}-S_{\mathrm{g}}^{\mathrm{res}}},
$$

with $P_{e}=8.73 \times 10^{5} \mathrm{~Pa}, m=-\frac{1}{2.89}$, and $S_{1}^{\text {res }}, S_{\mathrm{g}}^{\text {res }}$ the residual saturations defined previously.
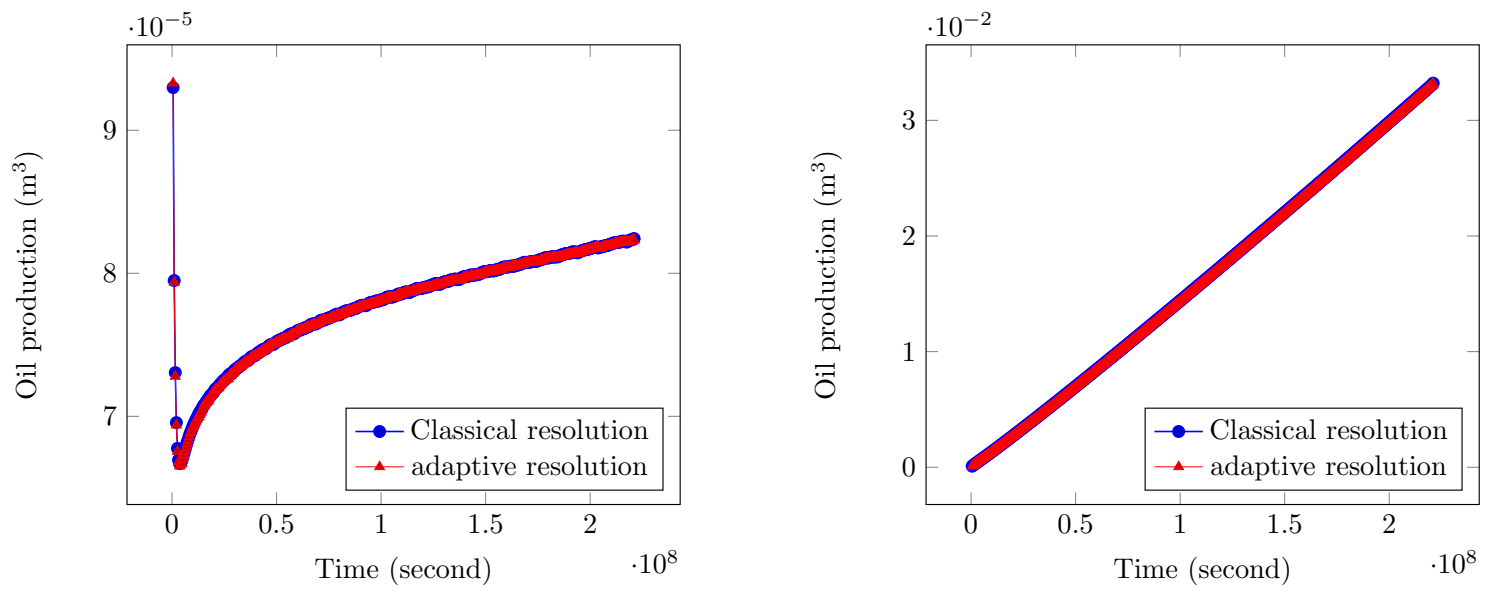

Figure 8: Rate (left) and cumulated rate (right) of oil production during the simulation, classical resolution vs. adaptive resolution for the test case of Section 5.3

Figure 3 shows the evolution of the mass balance violation measured as in (5.3), cf. Remark 4.7 We observe that the simulation based on the adaptive algorithm provides on average 2 digits accurate mass balance, while the classical algorithm based on fixed threshold algebraic stopping criterion yields on average 7 digits accuracy. As for the homogeneous case, we verify in Figure 8 that the adaptive resolution does not affect significantly the accuracy of the predicted oil production rate. Figure 9 shows the evolution of the liquid saturation and of the spatial estimator $4.19 \mathrm{a}$ ) at three different time steps. The error around the injection and production wells is again well detected, and the saturation front is accurately followed. As in the homogeneous case, we thus deem our estimators to be a good tool for adaptive mesh refinement.

The saved iterations from the linearization at each time step can be found in the left part of Figure 10 In the right part of this figure we show the cumulated number of Newton iterations during the simulation as a function of time steps; again a considerable gain in terms of the number of Newton iterations is achieved. In Figure 11 we compare the results obtained using the algebraic stopping criteria 4.22 , (5.1), and 5.4). At every time step and Newton iteration, the economy of the GMRes iterations using 

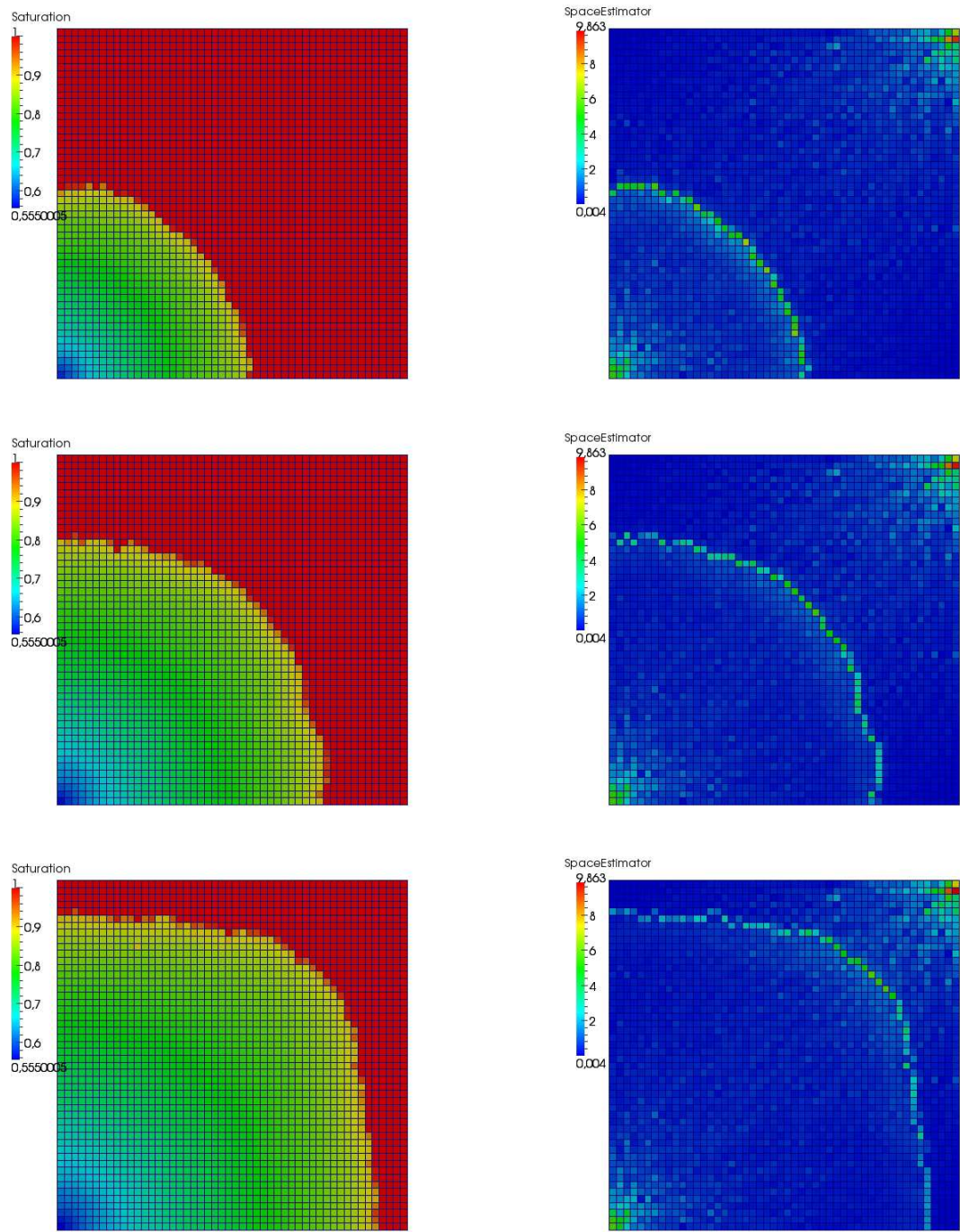

Figure 9: Liquid saturation (left) and distribution of the spatial estimators (right) for the adaptive resolution at times $5.2 \times 10^{7} \mathrm{~s}, 1.04 \times 10^{8} \mathrm{~s}$, and $1.6 \times 10^{8} \mathrm{~s}$, the test case of Section 5.3
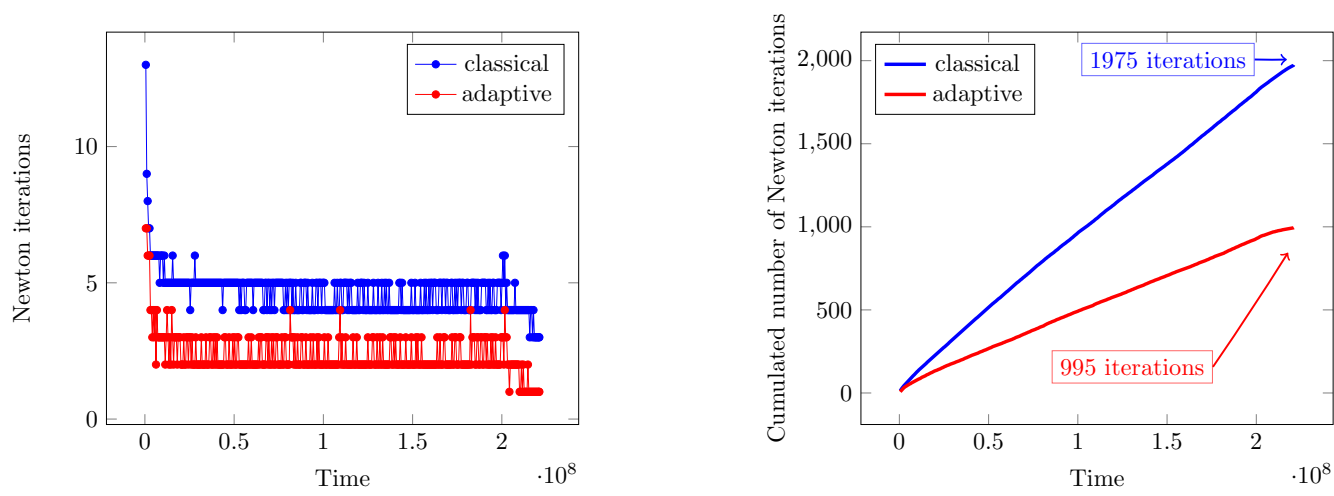

Figure 10: Newton iterations at each time step (left) and cumulated number of Newton iterations as a function of time (right) for the test case of Section 5.3. Average number of Newton iterations per time step: 5 iterations (classical), 2 iterations (adaptive) 

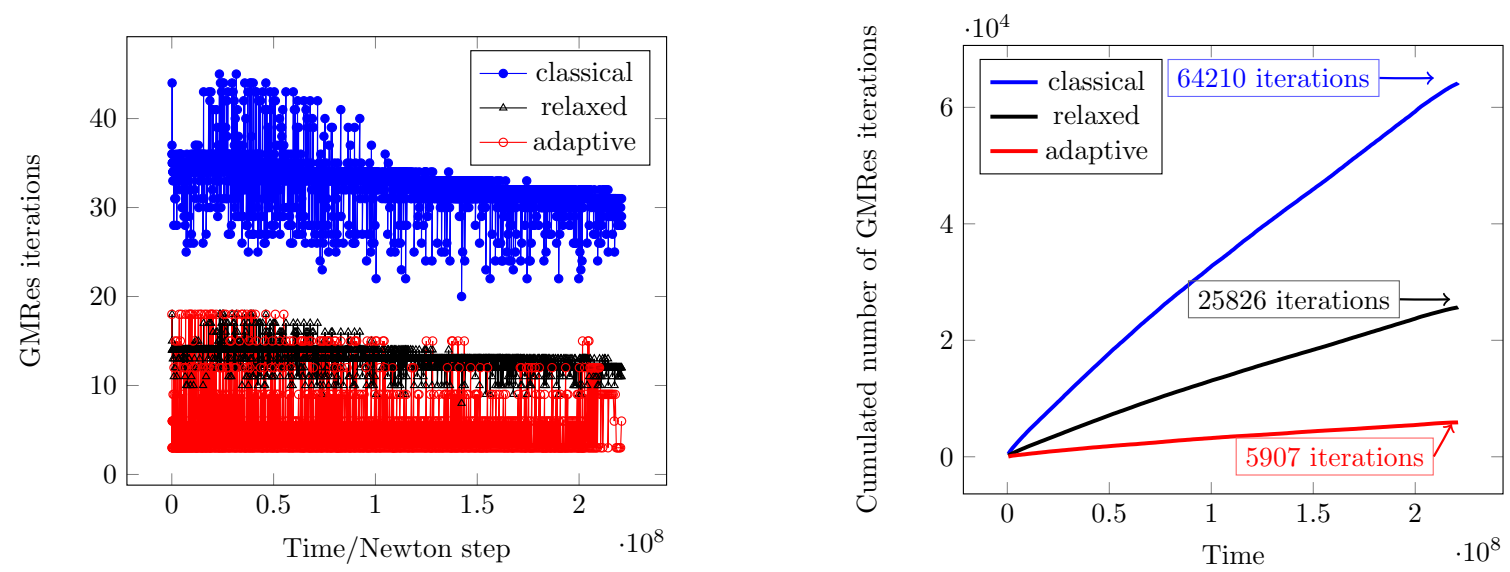

Figure 11: GMRes iterations at each time and Newton step (left) and cumulated number of GMRes iterations as a function of time (right) for the test case of Section 5.3. Average number of GMRes iterations per time and Newton iteration step: 33 iterations (classical), 13 iterations (relaxed), 6 iterations (adaptive). Average number of GMRes iterations per time step: 150 iterations (classical), 61 iterations (relaxed), 14 iterations (adaptive)

the stopping criterion 4.22 can be appreciated in the left part of Figure 11. In its right part, the overall gain is presented. We observe a gain factor for the adaptive criterion 4.22) of 10 compared with the classical criterion (5.1) and of 4.4 compared with the resolution using the relaxed criterion 5.4 These results are consistent with those found in the homogeneous case.

\section{References}

[1] I. Aavatsmark, T. Barkve, Ø. Bøe, and T. Mannseth. Discretization on non-orthogonal, curvilinear grids for multiphase flow. In Proc. of the 4th European Conf. on the Mathematics of Oil Recovery, volume D, Røros, Norway, 1994.

[2] I. Aavatsmark, T. Barkve, Ø. Bøe, and T. Mannseth. Discretization on unstructured grids for inhomogeneous, anisotropic media. I. Derivation of the methods. SIAM J. Sci. Comput., 19(5):1700-1716, 1998.

[3] Y. Achdou, C. Bernardi, and F. Coquel. A priori and a posteriori analysis of finite volume discretizations of Darcy's equations. Numer. Math., 96(1):17-42, 2003.

[4] G. Acs and E. Farkas. General purpose compositional model. Society of Petroleum Engineers, 25(4):543-553, 1985.

[5] L. Agélas, D. A. Di Pietro, and J. Droniou. The G method for heterogeneous anisotropic diffusion on general meshes. M2AN Math. Model. Numer. Anal., 44(4):597-625, 2010.

[6] L. Agélas, D. A. Di Pietro, and R. Masson. A symmetric and coercive finite volume scheme for multiphase porous media flow problems with applications in the oil industry. In Finite volumes for complex applications V, pages 35-51. ISTE, London, 2008.

[7] B. Amaziane, M. Jurak, and A. Žgaljić Keko. An existence result for a coupled system modeling a fully equivalent global pressure formulation for immiscible compressible two-phase flow in porous media. J. Differential Equations, 250(3):1685-1718, 2011.

[8] S. N. Antontsev, A. V. Kazhikhov, and V. N. Monakhov. Boundary value problems in mechanics of nonhomogeneous fluids. North-Holland, Amsterdam, 1990. Studies in Mathematics and Its Applications, Vol. 22.

[9] T. Arbogast. The existence of weak solutions to single porosity and simple dual-porosity models of two-phase incompressible flow. Nonlinear Anal., 19(11):1009-1031, 1992.

[10] K. Aziz and A. Settari. Petroleum Reservoir Simulation. Applied Science Publisher, Ltd, London, 1979.

[11] S. Balay, J. Brown, K. Buschelman, V. Eijkhout, W. D. Gropp, D. Kaushik, M. G. Knepley, L. C. McInnes, B. F. Smith, and Zhang H. PETSc users manual. Technical Report ANL-95/11 - Revision 3.3, Argonne National Laboratory, 2012 .

[12] S. Balay, J. Brown, K. Buschelman, W. D. Gropp, D. Kaushik, M. G. Knepley, L. C. McInnes, B. F. Smith, and H. Zhang. PETSc Web page, 2012. http://www.mcs.anl.gov/petsc.

[13] S. Balay, W. D. Gropp, L. C. McInnes, and B. F. Smith. Efficient management of parallelism in object oriented numerical software libraries. In E. Arge, A. M. Bruaset, and H. P. Langtangen, editors, Modern Software Tools in Scientific Computing, pages 163-202. Birkhäuser Press, 1997.

[14] J. Bear. Dynamics of fluids in porous media. American Elsevier, New York, 1972. 
[15] M. Bebendorf. A note on the Poincaré inequality for convex domains. Z. Anal. Anwendungen, 22(4):751-756, 2003.

[16] Y. Brenier and J. Jaffré. Upstream differencing for multiphase flow in reservoir simulation. SIAM J. Numer. Anal., 28:685-696, 1991.

[17] F. Brezzi and M. Fortin. Mixed and hybrid finite element methods, volume 15 of Springer Series in Computational Mathematics. Springer-Verlag, New York, 1991.

[18] R. J. Brooks and A. T. Corey. Hydraulic properties of porous media. Hydrology Paper 3, Colorado State University, Fort Collins, 1964.

[19] C. Cancès, T. Gallouët, and A. Porretta. Two-phase flows involving capillary barriers in heterogeneous porous media. Interfaces Free Bound., 11(2):239-258, 2009.

[20] C. Cancès, I. S. Pop, and M. Vohralík. An a posteriori error estimate for vertex-centered finite volume discretizations of immiscible incompressible two-phase flow. Math. Comp., 83(285):153-188, 2014.

[21] A. L. Chaillou and M. Suri. Computable error estimators for the approximation of nonlinear problems by linearized models. Comput. Methods Appl. Mech. Engrg., 196(1-3):210-224, 2006.

[22] G. Chavent and J. Jaffré. Mathematical models and finite elements for reservoir simulation. North-Holland, Amsterdam, 1986. Studies in Mathematics and Its Applications, Vol. 17.

[23] Z. Chen. Degenerate two-phase incompressible flow. I. Existence, uniqueness and regularity of a weak solution. $J$. Differential Equations, 171(2):203-232, 2001.

[24] Z. Chen and R. Ewing. From single-phase to compositional flow: Applicability of mixed finite elements. Transport in Porous Media, 27:225-242, 1997.

[25] Z. Chen, G. Qin, and R. E. Ewing. Analysis of a compositional model for fluid flow in porous media. SIAM J. Appl. Math., 60(3):747-777, 2000.

[26] K. H. Coats. An equation of state compositional model. Society of Petroleum Engineers, 20(5):363-376, 1980.

[27] D. A. Di Pietro and S. Lemaire. An extension of the Crouzeix-Raviart space to general meshes with application to quasi-incompressible linear elasticity and Stokes flow. Math. Comp., 2014. Accepted for publication. Preprint hal00753660

[28] D. A. Di Pietro and M. Vohralík. A review of recent advances in discretization methods, a posteriori error analysis, and adaptive algorithms for numerical methods in geosciences. OGST Oil \& Gas Science and Technology, 2013. Accepted for publication.

[29] D. A. Di Pietro, M. Vohralík, and S. Yousef. Adaptive regularization, linearization, and discretization and a posteriori error control for the two-phase Stefan problem. Math. Comp., 2014. Accepted for publication. Preprint hal-00690862

[30] J. Douglas, Jr., R. E. Ewing, and M. F. Wheeler. The approximation of the pressure by a mixed method in the simulation of miscible displacement. RAIRO Anal. Numér., 17(1):17-33, 1983.

[31] J. Droniou. Finite volume methods for diffusion equations: introduction to and review of recent methods. M3AS Math. Models Meth. Appl. Sci., 2013. Submitted.

[32] M. G. Edwards and C. F. Rogers. Finite volume discretization with imposed flux continuity for the general tensor pressure equation. Comput. Geosci., 2:259-290, 1998.

[33] L. El Alaoui, A. Ern, and M. Vohralík. Guaranteed and robust a posteriori error estimates and balancing discretization and linearization errors for monotone nonlinear problems. Comput. Methods Appl. Mech. Engrg., 200(37-40):2782$2795,2011$.

[34] K. Eriksson and C. Johnson. Adaptive finite element methods for parabolic problems. IV. Nonlinear problems. SIAM J. Numer. Anal., 32(6):1729-1749, 1995.

[35] A. Ern and M. Vohralík. A posteriori error estimation based on potential and flux reconstruction for the heat equation. SIAM J. Numer. Anal., 48(1):198-223, 2010.

[36] A. Ern and M. Vohralík. Adaptive inexact Newton methods with a posteriori stopping criteria for nonlinear diffusion PDEs. SIAM J. Sci. Comput., 35(4):A1761-A1791, 2013.

[37] R. E. Ewing, B. A. Boyett, D. K. Babu, and R. F. Heinemann. Efficient use of locally refined grids for multiphase reservoir simulation. Society of Petroleum Engineers, 1989.

[38] R. Eymard, T. Gallouët, and R. Herbin. Finite volume methods, volume VII. In Handbook of numerical analysis, North-Holland, Amsterdam, 2000.

[39] R. Eymard, C. Guichard, R. Herbin, and R. Masson. Vertex-centred discretization of multiphase compositional Darcy flows on general meshes. Comput. Geosci., 16(4):987-1005, 2012.

[40] R. Eymard, R. Herbin, and A. Michel. Mathematical study of a petroleum-engineering scheme. M2AN Math. Model. Numer. Anal., 37(6):937-972, 2003.

[41] G. H. Golub and Z. Strakoš. Estimates in quadratic formulas. Numer. Algorithms, 8(2-4):241-268, 1994.

[42] Z. E. Heinemann. Using local grid refinement in a multiple-application reservoir simulator. Society of Petroleum Engineers, 1983.

[43] M. R. Hestenes and E. Stiefel. Methods of conjugate gradients for solving linear systems. J. Research Nat. Bur. Standards, 49:409-436 (1953), 1952. 
[44] R. D. Hornung and J. A. Trangenstein. Adaptive mesh refinement and multilevel iteration for flow in porous media. J. Comput. Phys., 136(2):522-545, 1997.

[45] R. Huber and R. Helmig. Node-centered finite volume discretizations for the numerical simulation of multiphase flow in heterogeneous porous media. Comput. Geosci., 4(2):141-164, 2000.

[46] P. Jiránek, Z. Strakoš, and M. Vohralík. A posteriori error estimates including algebraic error and stopping criteria for iterative solvers. SIAM J. Sci. Comput., 32(3):1567-1590, 2010.

[47] O. A. Karakashian and F. Pascal. A posteriori error estimates for a discontinuous Galerkin approximation of secondorder elliptic problems. SIAM J. Numer. Anal., 41(6):2374-2399, 2003.

[48] Z. Khalil and M. Saad. Degenerate two-phase compressible immiscible flow in porous media: the case where the density of each phase depends on its own pressure. Math. Comput. Simulation, 81(10):2225-2233, 2011.

[49] D. Kröner and S. Luckhaus. Flow of oil and water in a porous medium. J. Differential Equations, 55(2):276-288, 1984.

[50] D. Kröner and M. Ohlberger. A posteriori error estimates for upwind finite volume schemes for nonlinear conservation laws in multidimensions. Math. Comp., 69(229):25-39, 2000.

[51] Yu. Kuznetsov. Approximations with piece-wise constant fluxes for diffusion equations. J. Numer. Math., 19:309-328, 2011.

[52] Yu. Kuznetsov and S. Repin. New mixed finite element method on polygonal and polyhedral meshes. Russian J. Numer. Anal. Math. Modelling, 18:261-278, 2003.

[53] L. W. Lake. Enhanced Oil Recovery. Old Tappan, NJ; Prentice Hall Inc., 1989.

[54] M. R. Laydi and M. Ghilani. A general finite volume scheme for an elliptic-hyperbolic system using a variational approach. Zeitschrift für angewandte Mathematik und Physik ZAMP, 49(4):630-643, 1998.

[55] M. Mamaghani, G. Enchéry, and C. Chainais-Hillairet. Development of a refinement criterion for adaptive mesh refinement in steam-assisted gravity drainage simulation. Comput. Geosci., 15(1):17-34, 2011.

[56] R. H. Nochetto, A. Schmidt, and C. Verdi. A posteriori error estimation and adaptivity for degenerate parabolic problems. Math. Comp., 69(229):1-24, 2000.

[57] M. Ohlberger. A posteriori error estimate for finite volume approximations to singularly perturbed nonlinear convection-diffusion equations. Numer. Math., 87(4):737-761, 2001.

[58] G. S. H. Pau, A. S. Almgren, J. B. Bell, and M. J. Lijewski. A parallel second-order adaptive mesh algorithm for incompressible flow in porous media. Philos. Trans. R. Soc. Lond. Ser. A Math. Phys. Eng. Sci., 367(1907):4633-4654, 2009.

[59] G. S. H. Pau, J. B. Bell, A. S. Almgren, K. M. Fagnan, and M. J. Lijewski. An adaptive mesh refinement algorithm for compressible two-phase flow in porous media. Comput. Geosci., 16(3):577-592, 2012.

[60] L. E. Payne and H. F. Weinberger. An optimal Poincaré inequality for convex domains. Arch. Rational Mech. Anal., 5:286-292 (1960), 1960.

[61] P. H. Sammon. Dynamic grid refinement and amalgamation for compositional simulation. Society of Petroleum Engineers, 2003.

[62] S. Thomas. Récupération assistée du pétrole : panorama. OGST Oil \& Gas Science and Technology, 63(1):9-19, 2008.

[63] J. A. Trangenstein and Z. Bi. Multi-scale iterative techniques and adaptive mesh refinement for flow in porous media. Adv. Water Resour., pages 1175-1213, 2001.

[64] R. Verfürth. A posteriori error estimates for nonlinear problems. $L^{r}\left(0, T ; L^{\rho}(\Omega)\right)$-error estimates for finite element discretizations of parabolic equations. Math. Comp., 67(224):1335-1360, 1998.

[65] R. Verfürth. A posteriori error estimates for nonlinear problems: $L^{r}\left(0, T ; W^{1, \rho}(\Omega)\right)$-error estimates for finite element discretizations of parabolic equations. Numer. Methods Partial Differential Equations, 14(4):487-518, 1998.

[66] M. H. Vignal. Convergence of a finite volume scheme for an elliptic-hyperbolic system. M2AN Math. Model. Numer. Anal., 30(7):841-872, 1996.

[67] M. Vohralík. Residual flux-based a posteriori error estimates for finite volume and related locally conservative methods. Numer. Math., 111(1):121-158, 2008.

[68] M. Vohralík and M. F. Wheeler. A posteriori error estimates, stopping criteria, and adaptivity for two-phase flows. Comput. Geosci., 17(5):789-812, 2013.

[69] M. Vohralík and B. I. Wohlmuth. Mixed finite element methods: implementation with one unknown per element, local flux expressions, positivity, polygonal meshes, and relations to other methods. Math. Models Methods Appl. Sci., 23(5):803-838, 2013

[70] L. C. Young and R. E. Stephenson. A generalized compositional approach for reservoir simulation. Society of Petroleum Engineers, 23(5):727-742, 1983. 